\title{
CAMBIOS EN LAS DENOMINACIONES DE ORIGEN PROTEGIDAS DEL SECTOR DEL VINO EN ESPAÑA: MOVIMIENTOS ENTRE MUNDOS DE PRODUCCIÓN
}

\author{
Samuel Esteban Rodríguez \\ Grupo de Estudios de Desarrollo Territorial de la Universidad de Zaragoza (GEDETUZ) \\ samuelesteban@ono.com
}

\section{RESUMEN}

En este estudio se realiza un análisis comparativo de las Denominaciones de Origen Protegidas del sector del vino en España, entre la campaña vitivinícola de 2001-02 y la de 2012-13. Se ha utilizado como marco de trabajo la teoría de los mundos de producción para realizar una clasificación de las denominaciones, en base a sus características productivas y su mercado de destino. Para ello, se han utilizado indicadores cuantitativos de tipo de tecnología y tipo de mercado. Una vez realizada la clasificación se ha comprobado que se ajusta a los postulados del modelo teórico y se ha pasado a analizar los resultados de cada campaña y la evolución seguida por el sector. Entre 2001-02 y 2012-13 se registra un aumento de la especialización productiva y de la personalización del producto en un gran número de denominaciones de origen, que han pasado a formar parte del mundo de producción interpersonal. Pese a ello, entre las denominaciones que se mantienen dentro de un modelo industrial se observan ajustes dirigidos a mejorar su posición en la competencia en precios. El mundo del mercado y el mundo de la innovación se encuentran menos difundidos, pese a que los cambios legislativos han tendido a aumentar la diversidad dentro del sector.

Palabras clave: Denominación de Origen Protegida, mundos de producción, vino.

\section{ABSTRACT}

This study is a comparative analysis of the Spanish Protected Designation of Origin (PDO) wine-producing regions between the production years of 2001-2002 and 2012-2013.

Fecha de recepción: mayo 2016.

Fecha de aceptación: mayo 2017. 
The worlds of production theory was used as a framework for the classification of the different PDO regions based on their production characteristics and their target market. Use was made of a series of quantitative indicators related to technology and market type. Once the classification was made, it was verified whether this adjusted to the concepts of the theoretical model, and a subsequent analysis was performed on the results of each production year and the evolution taking place in the industry. Between 2001-2002 and 2013-2013 an increase was reported in product specialization and customization in a large number of PDO regions, which have shifted to become a part of the interpersonal world of production. In spite of this, among the PDO regions that had retained their model within the industrial world of production, adjustments were observed directed at improving their position by means of competitive pricing. The market world and the innovation world are less extended, despite legislative changes encouraging diversity within the industry.

Keywords: Protected Designation of Origin, worlds of production, wine.

\section{INTRODUCCIÓN}

Las certificaciones territoriales han cobrado importancia entre las regiones productoras de vino, alentadas por un mayor interés de los consumidores por el origen y la calidad del producto. Factores de índole local, como la climatología o el tipo de suelo y las prácticas de producción características de cada zona, tienen efecto sobre las cualidades del vino que se elabora en cada región, existiendo diferencias entre ellas. En el marco de la Unión Europea se encuentra reconocida la figura de la Denominación de Origen Protegida (DOP) para garantizar el origen íntegramente local de un producto de calidad diferenciada ${ }^{1}$.

Pese a que esta figura existe en España desde la aprobación del estatuto del vino de 1932, el mayor desarrollo se produce desde 1986, con la entrada de España en la Unión Europea. En la campaña vitivinícola de 1985-86 se contaba con un total de 29 denominaciones activas (MAGRAMA, 2001), mientras que en 2012-13 el total ascendía a 90 (MAGRAMA, 2014). Aunque prácticamente se ha triplicado el número de denominaciones, el ritmo de crecimiento no ha sido constante. Desde 1986 hasta la campaña de 2000-01 se creaba un promedio de 1,8 denominaciones por año y entre 2000-01 y 2012-13 se ha pasado a crear un promedio de 2,8. Este crecimiento se acentúa especialmente a partir de 2003, tras la aprobación de Ley de la Viña y el $\mathrm{Vino}^{2}$, pues al incorporarse diferentes niveles de protección dentro de la figura de la denominación de origen, como los vinos de pago o las denominaciones calificadas, se ha facilitado su difusión.

Como consecuencia, la diversidad de denominaciones ha aumentado, observándose diferencias tanto en los modos de producción como en los mercados a los que se dirige cada una de ellas: Por un lado existen denominaciones donde se produce vino predominantemente en pequeñas bodegas, abastecidas de sus propios viñedos (Ruiz, 2013). Por otro lado, se cuenta

1 Reglamento (CE) 1151/2012 del Parlamento Europeo y del Consejo, de 21 de noviembre de 2012, sobre regímenes de calidad de los productos agrícolas y alimenticios. Diario Oficial de la Unión Europea (L 343), 1-29.

2 Ley 24/2003, de 10 de julio, de la Viña y del Vino. Boletín Oficial del Estado (165), 27165-27179. 
con denominaciones donde bodegas de gran tamaño y cooperativas concentran la producción del vino, estas bodegas generalmente producen su vino a partir de uvas procedentes de una amplia red de viticultores (Sánchez et. al., 2010).

Junto a las diferencias en la forma de producción, se observan diferencias en el mercado de destino del producto final. Como plantean Climent y Sánchez (2015), existen denominaciones centradas en mercados elitistas, donde la personalización del producto tiene gran importancia. Entre los consumidores a los que se dirigen, priman las cualidades del producto por encima del precio a la hora de seleccionar un determinado vino. Así mismo, se cuenta con denominaciones que abastecen grandes mercados indiferenciados, donde la personalización del producto queda en segundo plano, en ellas cobra mayor importancia la venta de vino como producto alimenticio y la exportación a precios bajos. La forma en la que las compañías compiten entre sí varía en función del modelo productivo y el mercado de destino, este hecho se pone de manifiesto también al nivel de la denominación, pudiendo establecerse diferencias en cuanto al peso que el precio y la calidad tiene en cada caso (Esteban y Climent, 2015; Esteban, 2016).

Esta diversidad de situaciones propicia la identificación de grupos de denominaciones en función de su modelo predominante, en lo que respecta a la producción del vino y la comercialización. En este sentido, la teoría de los mundos de producción, planteada por Salais y Storper (1992), ofrece un marco de trabajo adecuado, pues plantea la existencia de diferentes tipologías de productos en función del cruce de 2 dimensiones principales: tipo de tecnología y tipo de mercado.

El tipo de tecnología diferencia entre producción especializada y estandarizada, en función del grado de generalización del conocimiento necesario para producir y de si en la producción aparecen economías de escala o no. Las tecnologías especializadas se relacionan con una producción llevada a cabo por un grupo reducido de expertos, mientras que las tecnologías estandarizadas con procedimientos automatizados. El tipo de mercado diferencia entre genérico y dedicado, correspondiéndose el primero con mercados predecibles, compuestos por un gran número de compradores anónimos, y el segundo con mercados personalizados, donde existe un reducido número de consumidores. Estas dimensiones tienen una clara correspondencia con la diversidad de situaciones presente en el sector del vino, por lo que resulta especialmente apropiada su utilización para definir los diferentes tipos de producción y comercialización.

Salais y Storper (1992), a partir de la combinación de tipo de tecnología y tipo de mercado, identifican la existencia de 4 'mundos de producción' que se observan en la realidad, estando cada uno de ellos caracterizado por unas convenciones de calidad y una forma de competencia específica. Los mundos de producción que plantean son: el interpersonal (especializado - dedicado), el del mercado (estandarizado - dedicado), el de la innovación (especializado - genérico) y el industrial (estandarizado - genérico). Una vez caracterizado el tipo de tecnología y el tipo de mercado de cada denominación, es posible ubicarla en un mundo de producción en un momento determinado.

La adscripción a uno u otro mundo de producción puede variar mediante cambios técnicos o de tipo de mercado (Murdoch y Miele, 1999). Es decir, puede analizarse la evolución de cada denominación a partir de su posición en el diagrama de mundos de producción a lo largo del tiempo y, de forma agregada, la del sector. Este ejercicio fue puesto en práctica por 
Sánchez et. al. (2010) para explicar la evolución de las denominaciones vinícolas de Castilla y León, interrelacionando cambios en el marco tecnológico y el tipo de mercado. Esteban (2016) realizó una clasificación de las denominaciones del conjunto de España en diferentes cortes temporales, incorporando en su estudio los efectos de la crisis.

Los cambios legislativos han propiciado una modificación de la composición por tipos de denominación, fundamentalmente por un incremento del peso de los pagos vitícolas. Resulta de interés realizar un análisis comparativo entre el momento actual y la situación previa a la aprobación de la ley de la viña y el vino, con el fin de valorar sus efectos. Así mismo, dado que se observan diferentes orientaciones entre las denominaciones españolas, es conveniente poder identificar grupos homogéneos, de cara a la realización de campañas específicas dirigidas a cada uno de ellos o para facilitar la creación de alianzas estratégicas entre denominaciones.

Por ello, utilizando como marco de trabajo la teoría de los mundos de producción, este estudio tiene como objetivo identificar tanto el mundo de producción de pertenencia de cada denominación, como los movimientos registrados en el conjunto de las denominaciones españolas, desde inicios de la década de 2000 hasta la actualidad. Se ha optado por adoptar una metodología cuantitativa, a partir de fuentes estadísticas normalizadas, pues facilita un tratamiento homogéneo de la información y disponer de una gradación de situaciones en cada corte temporal analizado.

Los indicadores de tipo de tecnología pivotan en torno a la relación viticultor - bodega y al tamaño medio de la explotación; los de tipo de mercado hacen referencia al tamaño medio de la marca de vino, como medida de personalización (Esteban, 2016). A partir del cruce de estos elementos, se realiza una clasificación de las denominaciones activas en la campaña vitivinícola de 2001-02 y 2012-13. La clasificación se ha validado de forma empírica, comprobando su coherencia con los postulados del modelo teórico en lo que respecta a la forma de competencia dominante en cada mundo de producción, precio y calidad. Una vez validados los resultados de la clasificación, se analiza cada corte temporal y se identifican los movimientos entre mundos de producción registrados en el periodo de estudio.

\section{MARCO TEÓRICO}

Salais y Storper (1992) plantean que a pesar de la variedad de productos existentes, el número de lógicas de producción es limitado, existiendo unos modelos ideales o 'mundos de producción' que pueden observarse en el mundo real. Estos modelos se derivan del cruce de 2 dimensiones principales, tipo tecnología y tipo de mercado. El tipo de tecnología puede ser especializada o estandarizada y el tipo de mercado genérico o dedicado. Los mundos de producción resultantes son 4: El mundo interpersonal, el mundo del mercado, el mundo de la innovación y el mundo industrial. Cada uno de estos mundos de producción se define tanto por su combinación de tecnología y mercado, como por sus convenciones. Las convenciones son prácticas comúnmente aceptadas por los diferentes actores de la actividad económica y varían en cada mundo de producción, entre ellas se incluye la forma de competencia, es decir, la relación que se establece entre precio y calidad dentro de cada uno de ellos. 


\section{II.1. Dimensiones de producto}

Desde el punto de vista de la teoría de los mundos de producción todos los productos están definidos por un marco tecnológico determinado y su mercado de destino.

\section{II.1.1.Tipo de tecnología}

El tipo de tecnología con el que se produce un artículo puede ser especializado o estandarizado. Las tecnologías especializadas se caracterizan por el predominio de economías de gama y encontrarse restringido el conocimiento necesario para producir a un reducido grupo de expertos. Las tecnologías estandarizadas, por el contrario, son propensas a la automatización, por lo que aparecen economías de escala en la producción. La competencia en precios tiene especial relevancia en los productos estandarizados, mientras que entre los especializados prima la calidad (Murdoch et al., 2000; Kirwan, 2006).

En entornos estandarizados las inversiones se dirigen hacia la automatización de procesos, por lo que se tiende a reemplazar trabajo por capital; por contra en entornos especializados, a causa de la baja automatización, los costes laborales tienen mayor peso (Salais y Storper, 1992). La introducción de maquinaria en la agricultura se ha dirigido hacia la reducción de estos costes, siendo el resultado dentro del sector del vino un aumento del tamaño medio de la explotación, es decir, de la superficie de viñedo gestionada por cada viticultor. Por ello, Climent y Sánchez (2015) lo toman en consideración como elemento de diferenciación de tipo de tecnología, relacionando especialización con pequeñas explotaciones y estandarización con explotaciones de gran tamaño.

En su estudio sobre Rioja, Salazar y Galve (2011) ponen de manifiesto el papel que tienen las cooperativas vinícolas a la hora de reducir los costes de transacción y generar economías de escala, característica propia de tecnologías estandarizadas. En Castilla y León, la creación de este tipo de bodegas de gran tamaño en la década de 1960 facilitó la automatización de procesos, introduciendo por ejemplo el envasado industrial de vinos, y aumentó la estandarización de la producción (Sánchez et al., 2010).

En la actualidad se ha generalizado la idea de que el vino se hace en la viña (Aparicio et al., 2008), a la vez que las bodegas buscan un producto homogénero y de alta calidad. El resultado es que se está produciendo un aumento del número de bodegas que cultivan sus propios viñedos (Sánchez et al., 2010), (Escalona et al., 2013; Ruiz, 2013). El reconocimiento de la figura de protección de los vinos de pago, que tiene una correspondencia directa con el modelo Château de viñedo-bodega, muestra esta tendencia. El avance de este modelo, en el que la producción de la uva queda en manos de un reducido grupo de expertos, se ha visto acompañado de una reducción de la agricultura a tiempo parcial, tal como pone de manifiesto Sánchez et al. (2010) para el caso de Castilla y León. Las pequeñas explotaciones han sufrido un retroceso, salvo aquellas que cuentan con viejos viñedos, muy apreciados por las bodegas (Sánchez et al., 2010).

\section{II.1.2. Tipo de mercado}

El tipo de mercado diferencia entre mercados dedicados y mercados genéricos. Los mercados dedicados se relacionan con una demanda incierta, pues en ellos predomina la perso- 
nalización del producto. Los mercados genéricos se relacionan con la presencia de un amplio número de consumidores anónimos y una demanda predecible. En la competencia entre empresas la diferenciación juega un papel importante en los mercados dedicados, los productos genéricos tienden a ser de similares características entre si. En los mercados dedicados las compañías deben ajustarse al corto plazo, mientras en los genéricos se puede realizar una planificación de la producción en el medio y largo plazo, lo cual, por otro lado, facilita su estandarización ya que ambas dimensiones se encuentran interrelacionadas.

Kirwan (2006) en su estudio sobre los mercados de agricultores destaca la importancia del trato directo entre productores y consumidores en los mercados dedicados. Salais y Storper (1992) plantean que el caso extremo de dedicación es la personalización total del producto, en este sentido tanto Murdoch et al. (2000) como Kirwan (2006) ponen de manifiesto que en mercados dedicados la oferta de producto es limitada, es decir, que se dirigen a un reducido grupo de consumidores. En el extremo opuesto se encuentran los productos comercializados a través de largas cadenas de distribución, que se corresponden con mercados genéricos (Stræte, 2008), en este grupo se incluye la venta de vino en grandes supermercados (Adinolfi et al., 2011). Es por ello que la presencia de marcas de vino de gran tamaño, necesarias para abastecer este tipo de cadenas de distribución, se vincula con mercados genéricos (Esteban, 2016). En este mismo sentido, Sánchez et al. (2010) considera como elemento característico de un aumento de la dedicación la reducción del tamaño de la serie.

\section{II.2. Los mundos de producción}

El resultado de la combinación de tipo de tecnología (especializada-estandarizada) y tipo de mercado (genérico-dedicado) define los diferentes mundos de producción (Figura 1).

Figura 1

DIAGRAMA DE MUNDOS DE PRODUCCIÓN

\begin{tabular}{|c|c|c|c|c|}
\hline & & Economías de gama & Economías de escala & \\
\hline \multirow{2}{*}{ 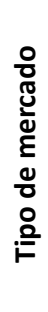 } & 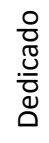 & $\begin{array}{c}\text { Mundo } \\
\text { interpersonal }\end{array}$ & $\begin{array}{l}\text { Mundo del } \\
\text { mercado }\end{array}$ & 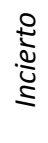 \\
\hline & 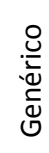 & $\begin{array}{l}\text { Mundo de la } \\
\text { innovación }\end{array}$ & $\begin{array}{l}\text { Mundo } \\
\text { industrial }\end{array}$ & $\begin{array}{l}\frac{0}{0} \\
\frac{d}{0} \\
\frac{d}{2} \\
\frac{0}{2}\end{array}$ \\
\hline & & Especializada & Estandarizada & \\
\hline & & \multicolumn{2}{|c|}{ Tipo de tecnología } & \\
\hline
\end{tabular}

Adaptado de Salais y Storper (1992). 


\section{II.2.1. Mundo interpersonal}

Este mundo de producción se corresponde con productos especializados-dedicados, se trata de productos personalizados que son fabricados a pequeña escala y donde la calidad tiene un peso fundamental (Salais y Storper, 1992; Murdoch 2000). En este mundo de producción el consumidor busca unas cualidades determinadas del producto, siendo estas el motivo principal de elección, por encima del precio. En el contexto de la nueva cultura del vino, el mundo interpersonal está integrado por bodegas que comercializan un producto de alta calidad, orientado a mercados elitistas (Sanchez et al., 2010).

\section{II.2.2. Mundo del mercado}

El mundo del mercado se corresponde con productos estandarizados y mercados dedicados, es decir, se trata de productos en cuya producción aparecen economías de escala y que se orientan a un nicho de mercado reducido. Existe pues cierta dualidad en este mundo de producción: por un lado las compañías deben cumplir con los requisitos de calidad y las cualidades que determina el nicho de mercado y, por otro lado, la tendencia hacia la automatización incide en una fuerte competencia en precios entre empresas.

Salais y Storper (1992) ponen de manifiesto que la tensión entre estandarización y dedicación suele resolverse en otros sectores industriales reduciendo costes a través de la externalización y la deslocalización. Sin embargo, la naturaleza de la propia denominación de origen limita las posibilidades de realizar este tipo de ajustes, por lo que el número de denominaciones en este mundo de producción es escaso (Esteban, 2016). El mundo del mercado en el sector del vino se relaciona con bodegas de gran tamaño que participan en concursos de vino y producen series diferenciadas (Sánchez et al., 2010) dentro de un entorno dominado por la competencia en precios (Esteban y Climent, 2015).

\section{II.2.3. Mundo de la innovación}

Este mundo de producción se corresponde con productos especializados-dedicados. Se trata de productos producidos por un reducido grupo de especialistas que se orientan a mercados de gran tamaño, en otros sectores se relaciona con el desarrollo de nuevos productos. El vino es un producto tradicional, en él, este mundo de producción se vincula con bodegas y denominaciones donde predomina del modelo château y la producción de vinos de calidad comercializados en series homogéneas para abastecer mercados de gran tamaño (Esteban, 2016). Este modelo de producción es frecuente en otros países como Italia donde en torno a un $79 \%$ de los vinos especializados se comercializan en supermercados (Adinolfi et al., 2011).

\section{II.2.4. Mundo industrial}

En el mundo industrial se producen artículos con tecnologías estandarizadas que se dirigen a mercados genéricos, se trata de artículos de gran consumo que tienen como destino un mercado grande y predecible. La fabricación de estos productos se encuentra generalizada y 
cuenta con alto grado de automatización, se pueden producir en masa por parte de diferentes compañías. Sus cualidades están bien definidas y son alcanzables mediante estándares industriales, por lo que la competencia entre empresas se centra en el precio. En el sector del vino este mundo de producción se relaciona con la presencia de grandes bodegas y cooperativas que producen vino para su venta en mercados de gran tamaño.

La etapa de industrialización y el trasvase de población del campo a la ciudad propició la aparición de grandes bodegas industriales para poder abastecer la demanda de vino como producto alimenticio de los mercados urbanos (Sánchez et al., 2010). Dentro de este mundo de producción la competencia tiende a estar centrada en el precio, lo cual también se ha puesto de manifiesto para el caso de las áreas productoras (Esteban y Climent, 2015), cabiendo relacionar la exportación de vino a bajo coste con este mundo de producción. Las denominaciones ubicadas en el mundo industrial se caracterizan por la producción de vino en bodegas de gran tamaño que facilitan la obtención de economías de escala, lo cual les permite abastecer mercados de gran tamaño y competir en precios (Esteban y Climent, 2015).

\section{II.3. Formas de competencia}

Las prácticas de producción, comercialización y negociación difieren en función del cruce de tipo de tecnología y mercado, es decir, existen convenciones diferentes en cada mundo de producción. Entre estas convenciones destaca la forma de competencia entre compañías, variando el peso que se otorga al precio y la calidad en cada mundo de producción (Figura 2). En los mundos de producción estandarizados, donde las cualidades requeridas se pueden alcanzar mediante procedimientos industriales, la competencia en precios es mayor, por contra en entornos especializados la calidad y la reputación de la marca tiene un papel más importante (Salais y Storper, 1992; Murdoch et al., 2000). En los mercados dedicados, personalizados, las cualidades específicas del producto cobran gran importancia, jugando la calidad diferenciada un importante rol dentro de la forma de competencia en este tipo de mercados, por encima del que juega en mercados genéricos, especialmente dentro del mundo industrial (Salais y Storper, 1992; Cazals, 2012).

Figura 2

MODELO DE COMPETENCIA PREDOMINANTE EN CADA MUNDO DE PRODUCCIÓN

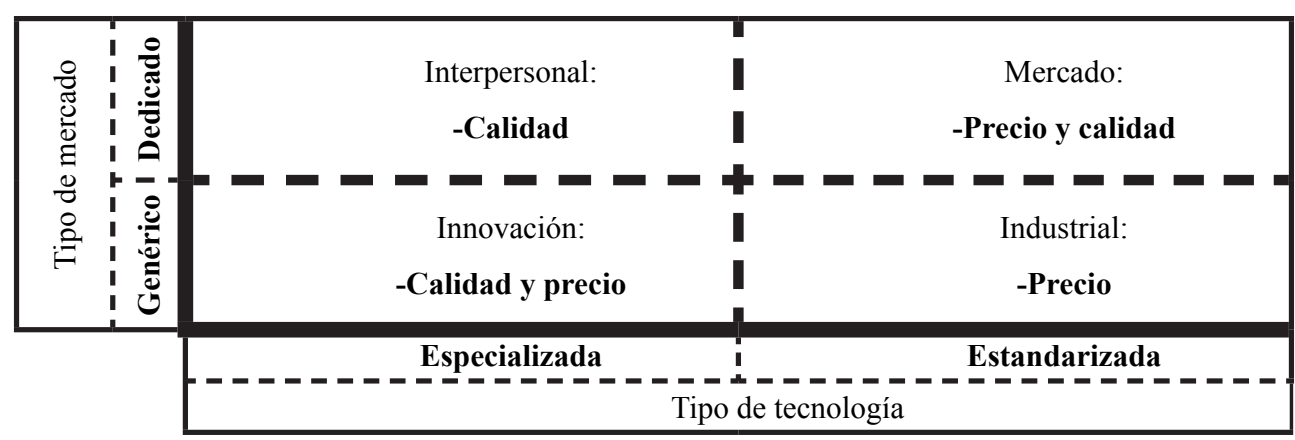

Adaptado de Salais y Storper (1992: 172) y Cazals (2012: 1187) 
Como consecuencia de las consideraciones previas, desde un punto de vista teórico, cabe identificar el tipo de competencia dominante dentro de cada mundo de producción: en el mundo interpersonal es la alta calidad el elemento fundamental, en el mundo del mercado la presencia de tecnologías estandarizadas centra la competencia en el precio, pero manteniendo los estándares de calidad del nicho de mercado. En el mundo de la innovación las tecnologías especializadas determinan que la competencia se centre en la calidad, jugando el precio un papel menor. En el mundo industrial, dado que todos los productos cuentan con una calidad y unas cualidades similares, la competencia se centra en el precio.

\section{II.4. Movimientos entre mundos de producción}

Los cambios en la forma en la que se produce un producto o en el mercado de destino generan variaciones en el mundo de producción de pertenencia. Estas modificaciones implican cambios en las convenciones de calidad y la forma de competencia (Salais y Storper, 1992). El movimiento entre mundos de producción puede interpretarse como la adaptación de un sector a las nuevas convenciones que demanda el mercado, suponiendo esto una forma de innovación (Stræte, 2004). La adaptación a estos cambios es fundamental para el éxito de un sector a lo largo del tiempo (Lindkvist y Sánchez, 2008).

Storper y Salais (1997) resaltan los desplazamientos en sentido horizontal y vertical dentro del diagrama de mundos de producción. Estos movimientos pueden ser por cambios técnicos o por cambios en el tipo de mercado. Los primeros, en la industria, generalmente suponen el paso desde el mundo de la innovación al mundo industrial, cuando madura un producto. Los segundos suelen ser desplazamientos del mundo industrial al del mercado causados por una demanda más personalizada (Murdoch y Miele, 1999). Junto a estos movimientos pueden registrarse otros, siendo los menos frecuentes los movimientos en diagonal.

En el trabajo de Sánchez et al. (2010) sobre los movimientos entre mundos de producción que se han registrado dentro del sector en Castilla y León, se expone como desde una primera etapa caracterizada por la producción a baja escala y pequeños mercados de proximidad, el sector del vino pasa a situarse en el mundo industrial en el periodo del desarrollismo. Este cambio fue motivado por el éxodo rural, que supuso una ampliación de la distancia entre productores y consumidores, lo que facilitó la aparición de grandes bodegas para el suministro de vino a los incipientes mercados urbanos. Posteriormente, algunas bodegas de gran tamaño comenzaron a participar en concursos de vinos y a poner en valor las cualidades del producto, segmentando su producción, lo que desde el punto de vista de la teoría de los mundos de producción supuso un cambio del mundo industrial al del mercado. El desarrollo de la nueva cultura del vino, centrada en la calidad del producto, ha originado un nuevo cambio, con un giro hacia el mundo interpersonal elitista (Sánchez et al., 2010). En este mundo de producción el vino se demanda en función de sus cualidades, adquiridas mediante unas condiciones determinadas de producción.

\section{METODOLOGÍA}

El esquema de trabajo planteado (Figura 3) se dirige a identificar el mundo de producción de pertenencia de las denominaciones de origen de vino en España entre la década de 2000 (campaña de 2001-02) y la actualidad (campaña de 2012-13), para poder analizar su 
evolución y valorar el efecto de la ley de la viña y el vino de 2003. Para poder cumplir con el objetivo propuesto, es necesario identificar el tipo de tecnología y el tipo de mercado de cada denominación en cada momento. Dado que los resultados de la clasificación han de ser comparables tanto entre denominaciones como a lo largo del tiempo, se han de utilizar indicadores homogéneos y cuyo procedimiento de obtención se haya mantenido constante, por lo tanto es preferible optar por fuentes estadísticas normalizadas.

Figura 3

ESQUEMADE TRABAJO

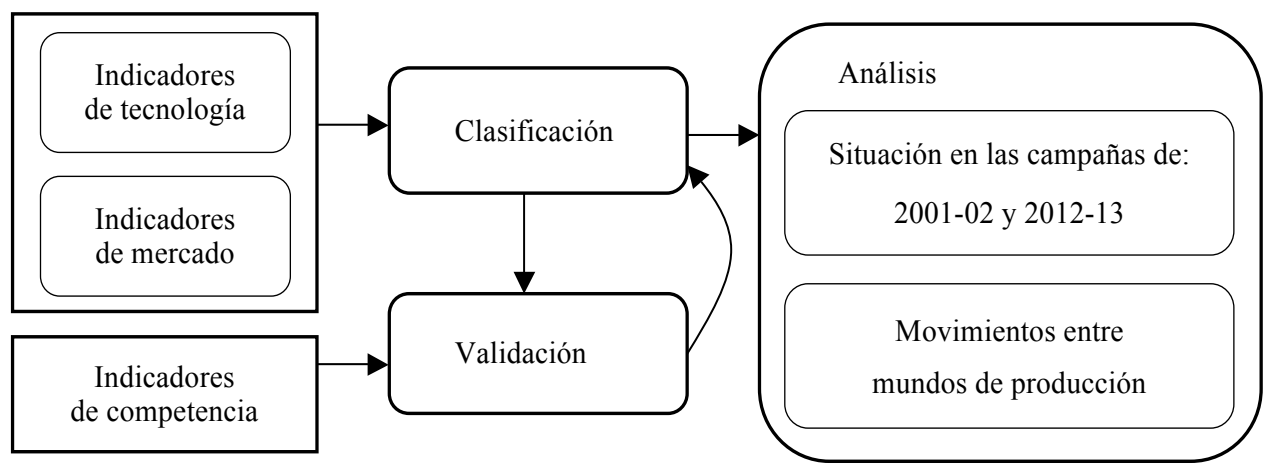

Una vez identificado el mundo de producción de pertenencia de cada denominación en cada campaña, a partir de indicadores de tipo de tecnología y tipo de mercado, es oportuno validar la coherencia de la clasificación realizada con los postulados del modelo teórico. Es posible comprobar que la forma de competencia, dentro de cada mundo de producción resultante de la fase de clasificación, se ajusta a los postulados teóricos. Para realizar esta tarea es necesario disponer de un indicador de calidad diferenciada y de otro de precio, con el fin de poder realizar las oportunas verificaciones.

Comprobado el modelo de clasificación, los resultados de cada campaña permiten estudiar la distribución de tipologías de denominación en cada corte temporal, siendo posible observar las denominaciones que integran cada categoría en cada momento. El análisis de movimientos entre mundos de producción se realiza en una fase posterior, a partir de la comparación de los resultados obtenidos en diferentes campañas, con el fin de identificar las denominaciones que se han mantenido estables y las que han cambiado de mundo de producción.

\section{III.1. Definición de indicadores}

\section{III.1.1. Tipo de tecnología}

Tomando en consideración la propensión hacia las economías de escala y el grado de generalización del conocimiento necesario para producir, en el sector del vino pueden considerarse 2 aspectos principales a la hora de diferenciar entre tecnologías especializadas y 
estandarizadas. Por un lado el tamaño de la explotación vitícola y por otro la cercanía al modelo château, en el que cada bodega se abastece por uvas de un único viticultor, o al modelo de las grandes bodegas cooperativas, en el que la uva procede de un elevado número de viticultores.

La superficie media cultivada por cada viticultor es reflejo de la estructura de las explotaciones agrícolas de la denominación. La mecanización de las labores agrarias ha permitido un aumento del tamaño medio de la explotación. Este proceso, como se ha comentado, ha modificado la relación entre trabajo y capital, siendo esta una de las funciones que proponen Salais y Storper (1992) de cara a maximizar en el extremo estandarizado del eje. En consecuencia, como plantean Esteban y Climent (2015), la utilización del indicador de superficie por viticultor resulta adecuada para diferenciar entre tecnologías especializadas (valores bajos) y estandarizadas (valores altos).

El número de viticultores por bodega permite establecer una gradación continua entre el modelo de los pagos vitícolas (Château) y el de las grandes cooperativas. El primer modelo se relaciona con una mayor especialización y el segundo con una mayor estandarización (Sánchez et al., 2010). Los pagos vitícolas centran su producción en inputs de uva procedentes de viñedos controlados por la propia bodega, de cara a buscar una producción homogénea, centrada en la calidad (Ruiz, 2013). Por lo tanto, en los pagos, la producción de la uva queda en manos de un número reducido de expertos. Frente a este modelo se encuentra el de las cooperativas, donde el suministro se lleva a cabo por parte de un gran número de viticultores, lo que reduce los costes de transacción y favorece la aparición de economías de escala (Salazar y Galve, 2011). La aparición de economías de escala se relaciona con una tecnología estandarizada, mientras que la producción por parte de un limitado número de especialistas se relaciona con tecnologías especializadas (Salais y Storper, 1992). Como resultado, la relación existente entre el número de viticultores y el de bodegas facilita la distinción entre tipos de tecnología, correspondiéndose valores bajos de viticultores por bodega con especialización y altos con estandarización.

Estos indicadores se pueden combinar de cara a obtener una escala única de tipo de tecnología. A tal efecto, Esteban (2016) plantea utilizar el producto entre ambos, es decir, derivar un indicador de superficie por bodega. Número de viticultores por bodega y tamaño de la explotación se encuentran íntimamente relacionados, ya que es necesaria cierta cantidad de uva para abastecer una bodega. Por ello, dentro del modelo de viñedo-bodega las explotaciones tienden a ser mayores, frente al modelo de cooperativas, que se concibe como la asociación de varios viticultores, con gran presencia de pequeñas explotaciones. Esta dualidad favorece el uso de ambos indicadores combinados, para poder contar con un indicador sintético que permita establecer una diferenciación entre especialización y estandarización. Valores bajos de superficie por bodega se relacionan con especialización y valores altos con estandarización.

\section{III.1.2. Tipo de mercado}

El grado de diferenciación del producto es un elemento clave a la hora de discernir el tipo de mercado. Mientras en los mercados genéricos la demanda se encuentra constituida por un elevado número de consumidores anónimos, que demandan de forma estable artículos cuyas 
cualidades son bien conocidas (Murdoch et al., 2000), en mercados dedicados la personalización juega un papel importante y es mayor la incertidumbre respecto a la demanda (Salais y Storper, 1992). Por ello, las series dirigidas a abastecer mercados genéricos son grandes, mientras que son cortas cuando se ha de cubrir la demanda específica de un nicho de mercado compuesto por un reducido número de consumidores.

En su análisis acerca un grupo de denominaciones de Castilla León y Aragón, Climent y Sanchez (2015) utilizaron como indicador de grado de diferenciación el número de marcas de vino que produce cada bodega. Este indicador es muy adecuado para comparar contextos tecnológicos similares, sin embargo no ofrece información acerca del tamaño del mercado al que se dirige cada una de las marcas. Tanto Murdoch et al. (2000) como Kirwan (2006) hacen referencia al grado de anonimia de los compradores como elemento de diferenciación de tipos de mercado. A partir del indicador de diferenciación de número de marcas por bodega y el volumen comercializado se puede derivar el tamaño medio de la marca, lo cual sí permite contar con una estimación del tamaño del mercado de destino (Esteban, 2016).

Adinolfi et al. (2011) plantea que la venta de vino en supermercados constituye un ejemplo de mercado genérico; el abastecimiento de grandes cadenas de supermercados se lleva a cabo a partir de series de gran tamaño, lo cual refuerza el uso del indicador del tamaño medio de la marca como elemento de diferenciación. Tamaños de marca reducidos se relacionan con mercados dedicados, personalizados, mientras que tamaños de marca grandes se relacionan con mercados genéricos, compuestos por un gran número de consumidores anónimos.

\section{III.1.3. Forma de competencia}

La teoría de los mundos de producción plantea que en cada mundo de producción existe una relación diferente entre precio y calidad, estableciéndose diferentes modelos competitivos. Para poder validar la coherencia de la clasificación con los postulados del marco teórico es necesario disponer de indicadores que hagan referencia a ambos. Por un lado, para valorar la competencia en precios, se puede utilizar el precio medio del vino comercializado por cada denominación, correspondiéndose los valores bajos con un alto peso de la competencia en precios y viceversa.

A diferencia del precio, que puede considerarse una variable cuantitativa definida de forma objetiva, definir un indicador de calidad resulta más complejo, pues son múltiples las perspectivas que se pueden considerar a la hora de valorar la calidad de un producto. De cara a sintetizar los múltiples aspectos que pueden valorarse en el caso del vino, Ruiz (2013) en su análisis sobre los pagos manchegos utiliza resultados de catas que son publicados en anuarios de vinos. Climent y Sánchez (2015) utilizan como indicador de calidad diferenciada el número total de premios obtenidos en el concurso de vinos Bacchus. Para poder disponer de un indicador de reconocimiento de la calidad diferenciada, en relación con la producción, se ha optado por utilizar la ratio entre el volumen total de vino comercializado y el número de premios. Esta medida permite comparar denominaciones de diferentes tamaños (Esteban y Climent, 2015; Esteban, 2016), puntuaciones altas se corresponden con un alto peso de la competencia en calidad y viceversa. 


\section{III.2. Fuentes y tratamiento de la información}

Cada campaña vitivinícola, el Ministerio de Agricultura, Alimentación y Medio Ambiente facilita información referida al nivel de la denominación en la publicación de "Cifras y Datos de las Denominaciones de Origen Protegidas"3. La información que suministra abarca todo el periodo de estudio e incluye datos sobre superficie inscrita, número de bodegas, volumen de vino comercializado y valor económico total del vino comercializado por cada denominación. Esta fuente de datos por si misma permite el cálculo del indicador de superficie por bodega y del precio medio del hectolitro de vino en precios corrientes, a su vez es una fuente auxiliar a la hora de construir el indicador de tipo de mercado y el de calidad diferenciada (Tabla 1). El precio medio del hectolitro de vino, una vez calculado, ha sido deflactado y expresado en base 2011 para disponer de un indicador en valores constantes, comparables entre campañas. Para llevar a cabo esta operación se ha recurrido a la tabla de medias nacionales del índice de precios al consumo por clases, que facilita el Instituto Nacional de Estadística (INE) referida al vino ${ }^{4}$.

Tabla 1

CÁLCULO DE INDICADORES

\begin{tabular}{|l|l|l|l|}
\hline Dimensión & Indicador & Cálculo & Fuentes \\
\hline Tipo de tecnología & Superficie por bodega & $\begin{array}{l}\text { Superficie inscrita total (ha) / Nú- } \\
\text { mero de bodegas }\end{array}$ & MAGRAMA \\
\hline Tipo de mercado & $\begin{array}{l}\text { Tamaño medio de la } \\
\text { marca }\end{array}$ & $\begin{array}{l}\text { Promedio del volumen de vino co- } \\
\text { mercializado por bodega (hl)/ Nú- } \\
\text { mero medio de marcas por bodega }\end{array}$ & $\begin{array}{l}\text { MAGRAMA Y } \\
\text { ANUARIO }\end{array}$ \\
\hline Forma de compe- \\
tencia & Precio medio & $\begin{array}{l}\text { Valor económico total (€) / Volu- } \\
\text { men comercializado (hl) }\end{array}$ & $\begin{array}{l}\text { MAGRAMA E } \\
\text { INE }\end{array}$ \\
\cline { 2 - 5 } & $\begin{array}{l}\text { Premios por volumen } \\
\text { comercializado }\end{array}$ & $\begin{array}{l}\text { lo.000 * ( Número de premios en } \\
\text { concursos / Volumen de vino co- } \\
\text { mercializado) }\end{array}$ & $\begin{array}{l}\text { MAGRAMA Y } \\
\text { BACCHUS }\end{array}$ \\
\hline
\end{tabular}

MAGRAMA: Serie de cifras y datos de las denominaciones de origen del Ministerio de Agricultura, Alimentación y Medio Ambiente campañas 2001-02 y 2012-13

ANUARIOS: Anuario de vinos el país $2002^{5}$ y $2013^{6}$

BACCHUS: Tabla de premios del concurso Bacchus ${ }^{7}$ edición 2012

3 Publicación de "Cifras y datos de las DOP”: http://www.magrama.gob.es/es/alimentacion/temas/calidadagroalimentaria/calidad-diferenciada/dop/htm/cifrasydatos.aspx (Última consulta: 16/05/2016)

4 Índice de precios de consumo, resultados nacionales, vino:

http://www.ine.es/dyngs/INEbase/es/operacion.htm?c=Estadistica_C\&cid=1254736176802\&menu=resultados $\&$ secc=1254736194779\&idp=1254735976607 (Última consulta: 16/05/2016)

5 Anuario de los vinos El País. Madrid, ediciones El País, 2002, 396 p.

6 Anuario de los vinos El País. Madrid, ediciones El País, 2013, 350 p.

7 Tablas de premiados en cada edición del concurso Bacchus:

http://www.uec.es/bacchus/index.php/es/medallas (Última consulta: 16/05/2016) 
El número medio de marcas por bodega se puede obtener a partir de anuarios de vino. Se ha optado por el anuario de vinos El País dado que segmenta la información por denominación de origen. El valor del indicador es necesario estimarlo: El procedimiento es contabilizar el número total de marcas presentes en el anuario para cada denominación y ponerlo en relación con el número total de bodegas que facilitan información. Para derivar el tamaño medio de la marca es necesario combinar información del Ministerio de Agricultura con información del anuario. El cálculo es el resultado de dividir la ratio de volumen de vino comercializado por bodega, estimado a partir de información del ministerio, entre el número medio de marcas por bodega, estimado a partir de información del anuario. La equivalencia temporal entre la información del ministerio y el anuario de vino se ha establecido en base al año final de la campaña, que va de julio del año inicial a julio del año siguiente, pues el anuario se edita en enero.

El número de premios en concursos de vino, necesario para derivar el indicador de calidad diferenciada seleccionado, se ha agregado a partir de las tablas de premios del concurso Bacchus. Pese a que el concurso no cuenta con edición en 2001, en 2013 está integrado en el calendario de premios del Ministerio de Agricultura ${ }^{8}$, motivo por el cual se ha seleccionado. El procedimiento de cálculo es el siguiente: Para cada denominación se ha contabilizado el número de premios obtenido en todas las categorías, este valor se ha dividido entre el volumen total de vino comercializado. El resultado se ha expresado en premios por cada 10.000 hectolitros para facilitar su interpretación.

El Ministerio de Agricultura hasta la fecha no facilita información desglosada para las denominaciones con consejos reguladores comunes, estas son por un lado de Jerez y Manzanilla S.B. y por otro Málaga y Sierras de Málaga. Por lo tanto, estas denominaciones se han tratado como una unidad, referida al consejo.

Los indicadores planteados se han recogido en una tabla de datos. Para cada denominación y campaña se ha registrado el valor en el indicador de tecnología, el de mercado y los 2 de competencia. De forma adicional, de la publicación del ministerio se ha recogido el número de viticultores para facilitar la explicación de resultados. En lo que respecta a los indicadores destinados a verificar la clasificación cabe decir que cuentan con una compleción menor que los destinados a la clasificación (Tabla 2). Los datos disponibles permiten clasificar el 77,08\% de las unidades consideradas, un 80,36\% de las activas en la campaña de 2001-02 y un 75\% de las activas en 2012-13.

8 Resolución de 14 de diciembre de 2012, de la Dirección General de la Industria Alimentaria, por la que se publica el calendario de concursos de vinos que se celebrarán durante el año 2013 en el territorio nacional. BOE, 31 de diciembre de 2012, núm 314, p. 89767- 89771. URL: http://www.boe.es/boe/dias/2012/12/31/pdfs/BOE-A-201215810.pdf (Última consulta 10/05/2016) 
Tabla 2

GRADO DE COMPLECIÓN DE LOS INDICADORES

\begin{tabular}{|l|l|r|r|r|}
\hline Tipo de indicador & \multicolumn{1}{|c|}{ Nombre } & Campaña 2001-02 & Campaña 2012-13 & \multicolumn{1}{c|}{ Total } \\
\hline & Número de unidades & 56 & 88 & 144 \\
\hline Clasificación & Superficie por bodega & $98,21 \%$ & $100,00 \%$ & $99,31 \%$ \\
\cline { 2 - 5 } & $\begin{array}{l}\text { Tamaño medio de la } \\
\text { marca }\end{array}$ & $80,36 \%$ & $75,00 \%$ & $77,08 \%$ \\
\hline \multirow{2}{*}{ Verificación } & $\begin{array}{l}\text { Unidades con ambos } \\
\text { indicadores }\end{array}$ & $80,36 \%$ & $75 \%$ & $77,08 \%$ \\
& Precio medio & $46,43 \%$ & $81,82 \%$ & $68,06 \%$ \\
\cline { 2 - 5 } & $\begin{array}{l}\text { Premios por volumen } \\
\text { comercializado }\end{array}$ & & $63,64 \%$ & $38,89 \%$ \\
\hline
\end{tabular}

\section{III.3. Diseño y validación de la clasificación}

\section{III.3.1. Elementos de clasificación}

Los mundos de producción se definen como el cruce de tipo de tecnología y de tipo de mercado, por lo que es preciso establecer un punto de ruptura que diferencie especialización de estandarización y otro que permita diferenciar mercados genéricos de mercados dedicados. Salais y Storper (1992) plantean que el punto de bifurcación entre mundos de producción se determina por el cambio en las convenciones de calidad dominantes, sin embargo no se cuenta en la actualidad con criterios objetivos suficientes para ubicarlo de forma precisa. Para solucionar esta cuestión, Climent y Sánchez (2015) utilizaron como referencia valores medios. Esteban y Climent (2015) adoptaron como punto de corte la mediana, para diferenciar las mitad mas especializada de la mas estandarizada y la mitad que se dirige a un mercado más genérico de la que lo hace a uno más dedicado.

El uso de medidas estadísticas de tendencia central permite establecer los puntos de corte necesarios para realizar la clasificación. Para cada eje, se ha utilizado el entorno de la mediana del conjunto de la serie de datos, con el fin de utilizar los mismos criterios para clasificar todas las campañas. La mediana del indicador de superficie por bodega es de 98,14 $\approx 100$ ha/bodega y la del tamaño de la marca de $332,3 \approx 300 \mathrm{hl} /$ marca. Estos son los valores que se han utilizado como referencia de la clasificación (Tabla 3).

Denominaciones con un valor en el indicador de superficie por bodega inferior o igual a 100 ha/bodega se corresponden con tecnologías especializadas, por encima de 100 con tecnologías estandarizadas. Mercados dedicados se corresponden con un tamaño medio de la marca inferior o igual a $300 \mathrm{hl} /$ marca, mercados genéricos con tamaños de marca superiores a 300. Los mundos de producción resultantes son el fruto de combinar estas categorías: El mundo interpersonal (especializada-dedicado), mundo del mercado (estandarizada-dedicado), mundo de la innovación (especializada-genérico) y mundo industrial (estandarizadagenérico). 
Tabla 3

CRITERIOS DE CLASIFICACIÓN

\begin{tabular}{|l|l|l|l|}
\hline \multirow{2}{*}{ Dimensión } & \multicolumn{1}{|c|}{ Indicador } & \multicolumn{2}{|c|}{ Sentido } \\
\cline { 2 - 4 } & \multicolumn{1}{|c|}{ Valores bajos } & \multicolumn{1}{c|}{ Valores altos } \\
\hline Tipo de tecnología & $\begin{array}{l}\text { Superficie por bodega } \\
\text { (ha/bodega) }\end{array}$ & Especializado $<=100$ & Estandarizado $>100$ \\
\hline Tipo de mercado & $\begin{array}{l}\text { Tamaño medio de la } \\
\text { marca } \\
\text { (hl/marca) }\end{array}$ & Dedicado $<=300$ & Genérico $>300$ \\
\hline
\end{tabular}

\section{III.3.2. Verificación}

La teoría de los mundos de producción plantea la existencia de diferencias en las formas de competencia dentro de cada mundo de producción. Para validar la clasificación se ha analizado su correspondencia con los postulados propuestos en el marco teórico respecto a estas formas de competencia. Si la clasificación es coherente con el modelo deben existir diferencias en el precio medio y en el indicador de calidad entre los diferentes mundos de producción. El precio medio ha de ser más bajo donde predominan las tecnologías estandarizadas y más alto entre las especializadas, y, en segundo lugar, ligeramente más bajo en mercados genéricos que dedicados. El indicador de calidad diferenciada ha de ser mayor en mercados dedicados que genéricos, $\mathrm{y}$, en segundo lugar, mayor en entornos especializados que estandarizados.

Para verificar estos planteamientos se ha realizado una comparación de las medias de los indicadores de calidad planteados en cada mundo de producción. De forma adicional, siguiendo a Hair et al. (1999), se han validado los supuestos de independencia, normalidad y homogeneidad de varianzas necesarios para realizar un análisis de la varianza (ANOVA), utilizando como factor la clasificación realizada y como variable de respuesta el precio y el indicador de calidad (Tabla 4). El precio cumple con todos los supuestos, el indicador de calidad no; por ello se ha realizado un ANOVA para el indicador de precio (Tabla 5); el indicador de calidad se ha analizado mediante la prueba no paramétrica Kruskal-Wallis (Tabla 6).

Los resultados obtenidos validan la clasificación realizada, en lo que respecta a las formas de competencia en cada mundo de producción. Se han encontrado diferencias en cuanto a precio e indicador de calidad coherentes con el modelo teórico. En la clasificación realizada los precios medios más altos se encuentran en mundos de producción caracterizados por una tecnología especializada, siendo más bajos en aquellos que producen vino aplicando tecnologías estandarizadas (Tabla 7). Así mismo se observa un diferencial en lo que respecta al eje de mercado, siendo ligeramente superior el precio en mercados dedicados que genéricos. Estas diferencias son estadísticamente significativas. 
Cambios en las denominaciones de origen protegidas del sector del vino en España: movimientos entre ...

Tabla 4

PRUEBAS DE NORMALIDAD Y HETEROCEDASTICIDAD DE LOS INDICADORES DE PRECIO Y CALIDAD ${ }^{9}$

\begin{tabular}{|c|c|c|c|c|c|c|c|}
\hline & \multicolumn{2}{|c|}{ Estadístico } & Global & Interpersonal & Mercado & Innovación & Industrial \\
\hline \multirow{9}{*}{ 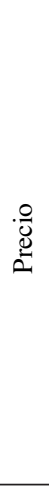 } & \multicolumn{2}{|l|}{ Número de casos } & 76 & 31 & 11 & 12 & 22 \\
\hline & \multicolumn{2}{|l|}{ Media } & 377,46 & 503,60 & 324,13 & 411,89 & 207,61 \\
\hline & \multicolumn{2}{|l|}{ Desviación típica } & 211,96 & 215,63 & 146,73 & 184,05 & 100,76 \\
\hline & \multirow{2}{*}{ Normalidad } & $\begin{array}{l}\text { Kolmogorov- } \\
\text { Smirnov }\end{array}$ & 0,78 & 0,86 & 0,74 & 0,76 & 0,56 \\
\hline & & Sig. & 0,57 & 0,45 & 0,65 & 0,62 & 0,92 \\
\hline & \multirow{4}{*}{ Homocedasticidad } & Levene & 2,15 & \multirow{4}{*}{\multicolumn{4}{|c|}{$\begin{array}{l}\text { Cumple con supuestos de normalidad y homogeneidad } \\
\text { de varianzas }\end{array}$}} \\
\hline & & gl1 & 3 & & & & \\
\hline & & $\mathrm{g} 12$ & 72 & & & & \\
\hline & & Sig. & 0,10 & & & & \\
\hline \multirow{9}{*}{ 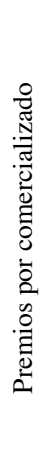 } & \multicolumn{2}{|l|}{ Número de casos } & 50 & 18 & 9 & 7 & 16 \\
\hline & \multicolumn{2}{|l|}{ Media } & 1,64 & 2,73 & 2,24 & 1,18 & 0,28 \\
\hline & \multicolumn{2}{|l|}{ Desviación típica } & 2,31 & 3,10 & 1,90 & 1,50 & 0,25 \\
\hline & \multirow[t]{2}{*}{ Normalidad } & $\begin{array}{l}\text { Kolmogorov- } \\
\text { Smirnov }\end{array}$ & 1,88 & 1,33 & 0,65 & 0,71 & 0,88 \\
\hline & & Sig. & 0,00 & 0,06 & 0,79 & 0,70 & 0,43 \\
\hline & \multirow{4}{*}{ Homocedasticidad } & Levene & 6,49 & \multirow{4}{*}{\multicolumn{4}{|c|}{$\begin{array}{l}\text { No cumple con supuestos de normalidad y } \\
\text { homogeneidad de varianzas }\end{array}$}} \\
\hline & & gl1 & 3 & & & & \\
\hline & & $\mathrm{g} 12$ & 46 & & & & \\
\hline & & Sig. & 0,00 & & & & \\
\hline
\end{tabular}

Tabla 5

DIFERENCIAS DE PRECIO ENTRE MUNDOS DE PRODUCCIÓN, TABLA DE ANOVA

\begin{tabular}{|l|l|r|r|r|r|r|}
\hline & & \multicolumn{1}{|c|}{ Suma de cuadrados } & \multicolumn{1}{c|}{ gl } & Media cuadrática & \multicolumn{1}{c|}{ F } & \multicolumn{1}{c|}{ Sig. } \\
\hline \multirow{3}{*}{ Precio } & Inter-grupos & $1.173 .481,191$ & 3 & $391.160,397$ & 12,825 &, 000 \\
\cline { 2 - 8 } & Intra-grupos & $2.196 .045,425$ & 72 & $30.500,631$ & & \\
\cline { 2 - 8 } & Total & $3.369 .526,616$ & 75 & & & \\
\hline
\end{tabular}

9 Dentro del mundo interpersonal, en el indicador de precio se han excluido las denominaciones de Prado Irache y Pago de Calzadilla por contar con valores atípicos, anormalmente altos, en la campaña de 2012-13. 
Tabla 6

DIFERENCIAS EN EL INDICADOR DE CALIDAD ENTRE MUNDO DE PRODUCCIÓN. ESTADÍSTICOS DE CONTRASTE, PRUEBADE KRUSKAL-WALLIS

\begin{tabular}{|c|c|c|c|}
\hline & Chi-cuadrado & gl & Sig. asintót. \\
\hline Premios por volumen comercializado & 23,924 & 3 &, 000 \\
\hline
\end{tabular}

Tabla 7

COMPARACIÓN DE INDICADORES DE COMPETENCIA EN CADA MUNDO DE PRODUCCIÓN

\begin{tabular}{|c|c|c|c|c|c|}
\hline \multicolumn{3}{|c|}{ Precio medio $(€)$ : } & \multicolumn{3}{|c|}{ Premios por comercializado: } \\
\hline Dedicado & 503,60 & 324,13 & Dedicado & 2,73 & 2,24 \\
\hline \multirow[t]{2}{*}{ Genérico } & 411,89 & 207,61 & Genérico & 1,18 & 0,28 \\
\hline & Especializado & Estandarizado & & Especializado & Estandarizado \\
\hline
\end{tabular}

En lo que respecta al indicador de calidad diferenciada utilizado, se observan mayores puntuaciones en los mundos de producción que se dirigen a mercados dedicados y menores en los genéricos. Donde predominan tecnologías estandarizadas también se observan puntuaciones superiores en el indicador de calidad que en entornos estandarizados, de nuevo, estas diferencias son coherentes con el modelo teórico.

Así mismo, se observa que el mundo interpersonal muestra mayores puntuaciones en el indicador de competencia en calidad y precios medios altos, lo que indica que la competencia en precios tiene un peso menor. En el mundo del mercado juega un importante papel la competencia en precios, así como la competencia en calidad. En el mundo de la innovación la competencia en precios es baja, pese a tener una mayor importancia que en el interpersonal, y el indicador de competencia en calidad también apunta a un alto peso de la misma. El mundo industrial es el que muestra una mayor competencia en precios y un menor peso de la competencia en calidad.

Estos resultados son similares a los obtenidos por Esteban (2016) a partir de los datos registrados en 3 campañas, sin embargo se observan ligeras diferencias en lo que respecta al peso de los premios en el mundo del mercado y el interpersonal. En el presente trabajo se ha encontrado un peso mayor de la competencia en calidad dentro del mundo interpersonal, tal como plantea el modelo teórico; en el trabajo de Esteban (2016) se encontraron puntuaciones similares de competencia en calidad en el mundo interpersonal y el mundo del mercado. Salvo esta cuestión, los resultados son muy similares en ambos casos, siendo también coherentes con las pruebas realizadas por Esteban y Climent (2015).

\section{III.4. Distribución e identificación de movimientos}

Una vez validada la metodología de clasificación, es posible pasar a analizar los resultados obtenidos en cada campaña y los movimientos registrados entre mundos de producción: 
El mundo de producción de pertenencia de cada denominación en cada campaña se ha analizado a través de tablas y mapas. En primer lugar se ha analizado la composición por mundos de producción en cada corte temporal, es decir, se ha comparado el porcentaje de denominaciones que integran cada modelo en 2001-02 y 2012-13. Los recorridos individuales de las denominaciones activas en todo el periodo se han analizado para poder identificar tendencias de cambio en el sector, para ello se han agregado a nivel nacional los diferentes movimientos registrados.

\section{RESULTADOS}

\section{IV.1. Distribución de los mundos de producción en cada corte temporal}

\section{IV.1.1. Campaña de 2001-02}

En la campaña de 2001-02 prácticamente la mitad de las denominaciones de origen de vino se encuentran ubicadas dentro del mundo industrial (Figura 4). El otro mundo de producción dirigido a mercados genéricos, el mundo de la innovación, es el segundo en importancia, con un $22,22 \%$ de las denominaciones. Las denominaciones orientadas a mercados dedicados no alcanzan un $30 \%$ del total, correspondiéndose un 17,78\% de ellas con el mundo interpersonal y un $11,11 \%$ con el del mercado. La situación en esta campaña refleja un predominio de tecnologías estandarizadas, con un $60 \%$ de las denominaciones, y de los mercados genéricos, con un $71,11 \%$.

Figura 4

MUNDOS DE PRODUCCIÓN DE LAS DENOMINACIONES DE ORIGEN DE VINO:

CAMPAÑA 2001-02, COMPOSICIÓN

\begin{tabular}{|c|c|c|c|}
\hline \multicolumn{3}{|c|}{ Porcentaje } & Número \\
\hline 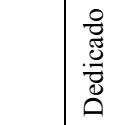 & $\begin{array}{c}\text { Mundo interpersonal: } \\
\qquad 17,78 \%\end{array}$ & $\begin{array}{l}\text { Mundo del mercado: } \\
\qquad 11,11 \%\end{array}$ & \\
\hline 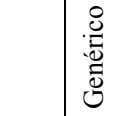 & $\begin{array}{l}\text { Mundo de la innovación: } \\
\qquad 22,22 \%\end{array}$ & $\begin{array}{l}\text { Mundo industrial: } \\
\text { 48,89\% }\end{array}$ & \\
\hline & Especializado & Estandarizado & $\begin{array}{ll}\text { Interpersonal } & \text { Mercado } \\
\text { Innovación } & \text { Industrial }\end{array}$ \\
\hline
\end{tabular}

Son frecuentes en la campaña de 2001-02 las denominaciones que producen vinos orientados a mercados indiferenciados, donde el peso de la competencia en precios es alto. En esta campaña la ley de la viña y el vino todavía no se había aprobado, por lo que la denominación de origen era la única figura presente. Las denominaciones activas en este corte temporal en 
su mayoría cuentan con una larga trayectoria y un alto grado de estandarización de sus procesos productivos, teniendo además alto peso el modelo cooperativista. Como resultado, el mundo industrial en esta campaña está fuertemente arraigado en un gran número de denominaciones, especialmente peninsulares (Figura 5). Entre ellas, son buenos ejemplos los casos de La Mancha, Cariñena, Rueda o Valencia, todas ellas denominaciones compuestas por bodegas abastecidas de una amplia red de viticultores y con un tamaño medio de la marca grande, de en torno a $700 \mathrm{hl} /$ marca.

El segundo grupo en importancia se corresponde con el compuesto por las denominaciones ubicadas dentro del mundo de la innovación. Se trata de denominaciones de gran prestigio que producen vinos en los que el reconocimiento de la calidad juega un papel importante. Entre ellas se encuentran en 2001-02 Rioja, Jerez o Rías Baixas, sin embargo, cada una cuenta con una posición diferenciada dentro del eje de tecnología. Jerez, por ejemplo, prácticamente se encuentra sobre el punto de ruptura entre especialización y estandarización, con 99,6 ha/bodega, mientras, Rioja cuenta con una puntuación de 37,2 ha/bodega. Por su parte la denominación de Cataluña, creada en 2001, se encontraba en esta campaña constituida por un reducido número de viticultores y un número relativamente alto de bodegas, por lo que también cabe ser considerada dentro del mundo de la innovación en sus primeras etapas de desarrollo. El arquetipo de denominación de origen del mundo de la innovación es Rioja, que cuenta con un alto grado de especialización y un tamaño de la marca grande, superior a los $500 \mathrm{hl} / \mathrm{marca}$.

El mundo interpersonal, compuesto por denominaciones caracterizadas por mercados personalizados y tecnologías especializadas, tiene un peso bajo en 2001-02. La orientación hacia mercados elitistas, donde la calidad es el elemento de elección primordial, era poco frecuente en esta campaña. Sin embargo, entre los vinos insulares si se observa un alto peso de este mundo de producción, tanto en el archipiélago canario como en el balear. En la España peninsular se han clasificado varias denominaciones dentro del mundo interpersonal, algunas como Bierzo o Cigales presentan bastante proximidad al punto de corte tanto en tipo de tecnología como en tipo de mercado. En este mundo de producción, destacan Priorato y Ribeira Sacra como los ejemplos más característicos: El tamaño medio de la marca en ellas es inferior a los $100 \mathrm{hl}$ y el nivel de especialización muy alto, con superficies por bodega inferiores a las 50 ha. El tipo de competencia que presentan se encuentra muy centrada en la calidad, no tanto en el precio, siendo en estas 2 denominaciones el precio medio superior a los $600 € /$ hl en la campaña de 2001-02, del entorno a los $1.200 € /$ hl en el caso de Priorato.

El número de denominaciones dentro del mundo del mercado en la campaña de 200102 es muy reducido, prácticamente anecdótico. En él se cuentan denominaciones como Ribera del Guadiana, fundada en 1996, la denominación insular de Lanzarote y otras como Bullas, Alella o Mondéjar. En este grupo la competencia en precios tiene un peso importante, por ejemplo en el caso de Bullas o Mondéjar ronda los 200-250€/hl, siendo estas denominaciones muy características de este mundo de producción en esta campaña. Las denominaciones de Alella y Lanzarote muestran unos precios medios más altos, así como una mayor proximidad al punto de corte de tipo de tecnología. 
Figura 5

MAPA DE MUNDOS DE PRODUCCIÓN DE LAS DENOMINACIONES DE ORIGEN EN 2001-02

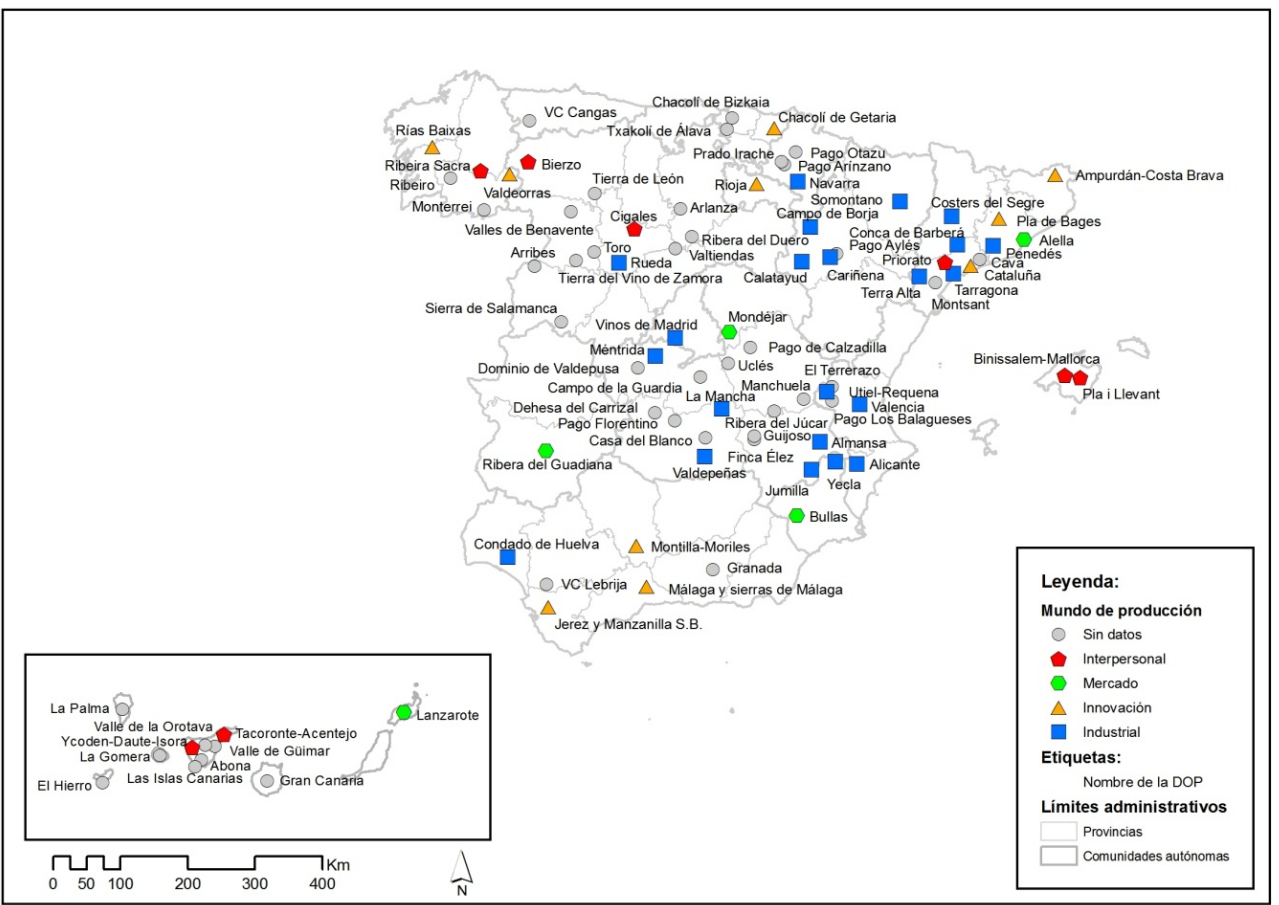

\section{IV.1.2. Campaña de 2012-13}

El mundo interpersonal es el más frecuente en la campaña de 2012-13, encontrándose integrado por un 43,94\% de las denominaciones clasificadas (Figura 6). El segundo mundo de producción en importancia es el mundo industrial, con un 25,76\% de las unidades analizadas. Mundo del mercado y mundo de la innovación cuentan con un 15,15\% de las denominaciones cada uno de ellos. Existe por tanto cierta dualidad en el sistema de denominaciones, con cerca de un $70 \%$ de las mismas operando en 2 contextos antagónicos: Por un lado el modelo de los vinos elitistas y por otro el de las grandes bodegas industriales que producen vinos poco diferenciados. El mundo interpersonal en la campaña de 2012-13 es dominante entre las denominaciones insulares, en las del noroeste de la península y entre los pagos vitícolas (Figura 7). En Galicia, País Vasco y en Castilla y León son numerosas las denominaciones que operan en mundos de producción especializados. Cabe diferenciar dentro de este mundo de producción entre denominaciones compuestas por pequeñas cooperativas de viticultores y denominaciones con una ratio de viticultores por bodega más baja. Entre las primeras se pueden citar Bierzo, Arribes o Abona, se trata de denominaciones que, pese a contar con una ratio de viticultores por bodega alta, presentan valores altos en el indicador de especialización y un intervalo de precios medio-alto. Entre las segundas por lo general el 
precio medio es ligeramente superior y destacan, por un lado, la denominación de Priorato o el Chacolí de Álava y, por otro, los pagos vitícolas. El modelo de los pagos fue puesto en marcha por la ley de la viña y en vino de 2003 y tiene una alta presencia dentro del mundo interpersonal en la campaña de 2012-13.

Figura 6

MUNDOS DE PRODUCCIÓN DE LAS DENOMINACIONES DE ORIGEN DE VINO: CAMPAÑA 2012-13, COMPOSICIÓN

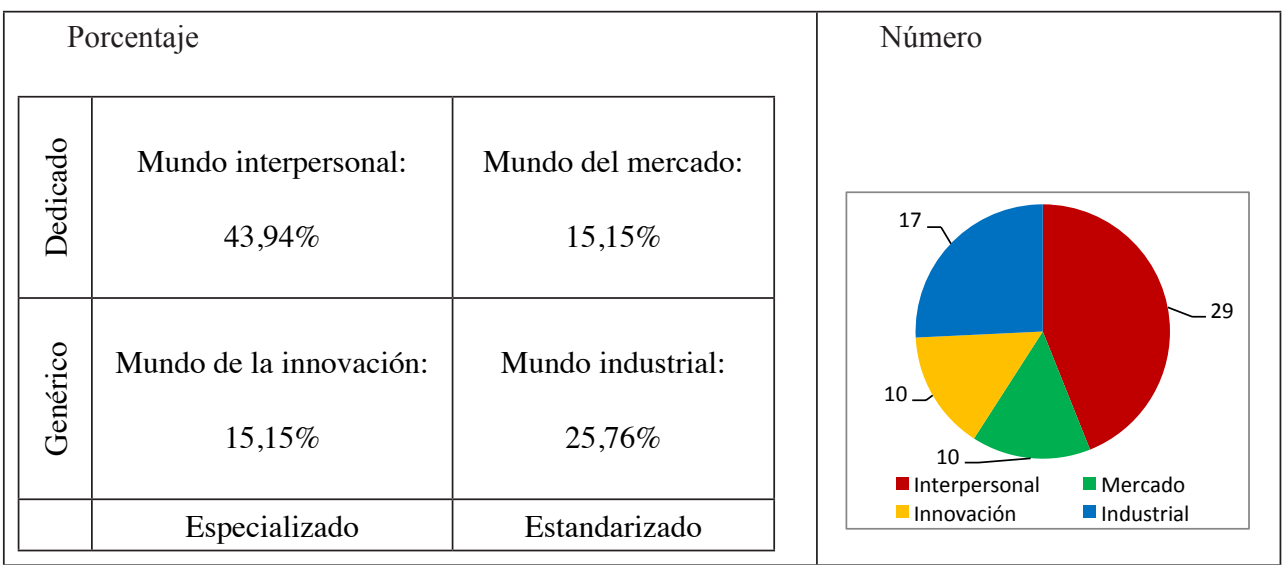

Figura 5

MAPA DE MUNDOS DE PRODUCCIÓN DE LAS DENOMINACIONES DE ORIGEN EN 2012-13
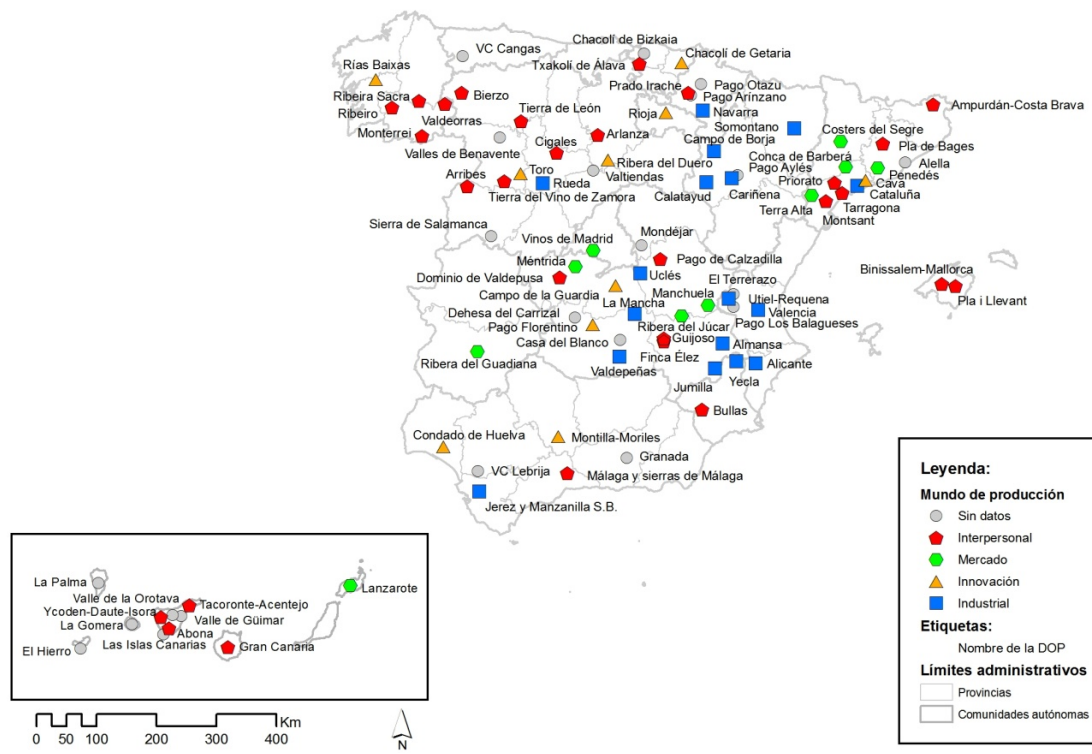

Arribes $\triangle \triangle^{\text {Toro }} \begin{gathered}\text { Ribera del Duero } \\ \text { Pago Ayles arbera }\end{gathered}$

Tierra del Vino de Zamora Calatayud Carinena Terra Alta Tarragona

Sierra de Salamanca Vinos de Madrid Mondejar

Mentrida Pago de Calzadilla

Dominio de Valdepusa

Campo de la Guardia La Mancha Manchuela 3 Utiel-Requena

Pago Florentino $\triangle$ Ribera del Júcar Pago Los Bal
Casa del Blanco
Guijoso Almansa

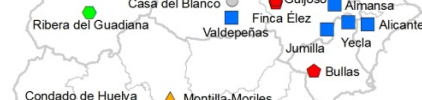

Condado de Huelva $\triangle$ Montilla-Moriles

L vC Lebrija Málaga y sierras de Mälaga

Jerez y Manzanilla S. B.

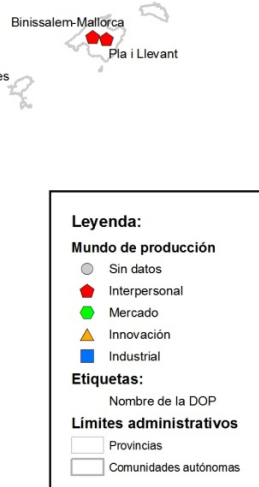


Las denominaciones integradas por bodegas industriales, que prioritariamente dirigen su producción hacia mercados indiferenciados, se concentran en el centro y este peninsular. En este mundo de producción destacan denominaciones como La Mancha, Valencia o Valdepeñas, en ellas la competencia en precios es alta, contando con precios relativamente bajos, de en torno a $250 € /$ hl. Existen algunas denominaciones como Navarra o Jerez que se encuentran muy próximas al punto de ruptura de tipo de tecnología, sin embargo otras como Jumilla, Yecla o Almansa no ofrecen lugar a dudas: sus puntuaciones en el indicador de estandarización son muy altas, al igual que las de tipo de mercado, con un tamaño medio de la marca grande.

Rioja, Ribera del Duero y Cava destacan dentro del mundo de la innovación, contando menos de 30 viticultores por bodega todas ellas. En estas denominaciones el indicador de tipo de tecnología ronda las 80 ha/bodega, el de tipo de mercado sobrepasa los 500 hl/marca, el de precio se sitúa por encima de la media y ofrecen buenos resultados en el indicador de reconocimiento de la calidad, siendo características todas ellas propias del mundo de la innovación. Es poco frecuente este mundo de producción en el conjunto de España, pese a ello, las denominaciones que lo integran comercializan volúmenes de vino muy importantes.

El mundo del mercado, al igual que el de la innovación, está poco extendido en el conjunto del país, pese a ello cuenta con presencia en la comunidad catalana, algunas denominaciones del centro del país y en Lanzarote. En la campaña de 2012-13 el tamaño medio de la marca, en todas las denominaciones clasificadas en este mundo de producción, es muy inferior al del punto de corte: Las diferencias son superiores a los $100 \mathrm{hl} / \mathrm{marca}$, lo cual indica una clara orientación hacia nichos de mercado reducidos. Méntrida, Ribera del Júcar o Vinos de Madrid son muy buenos ejemplos de este mundo de producción, contando con altos valores de estandarización y bodegas abastecidas por gran cantidad de viticultores, pero que a su vez cuentan con una producción diferenciada.

\section{IV.2. Evolución entre 2001-02 y 2012-13}

\section{IV.2.1. Cambios de composición}

Entre la campaña de 2001-02 y la de 2012-13, las tipologías de denominación presentes en el conjunto de España han variado considerablemente, observándose un aumento superior al 25\% en las denominaciones que operan dentro del mundo interpersonal (Figura 8). Existe una tendencia, por parte de muchas denominaciones, hacia una mayor especialización y una mayor dedicación, es decir: una búsqueda de mercados donde predomina la competencia en base a la calidad del producto. El mundo industrial, donde la competencia gira fundamentalmente en torno al precio, ha experimentado un fuerte retroceso, habiendo disminuido en un 23,13\% el número de denominaciones clasificadas en él. El mundo del mercado y el de la innovación han experimentado cambios de menor envergadura, inferiores al 10\% en ambos casos, con un sentido positivo en el mundo del mercado y un ligero descenso en el mundo de la innovación, lo cual confirma la tendencia hacia una mayor dedicación. 


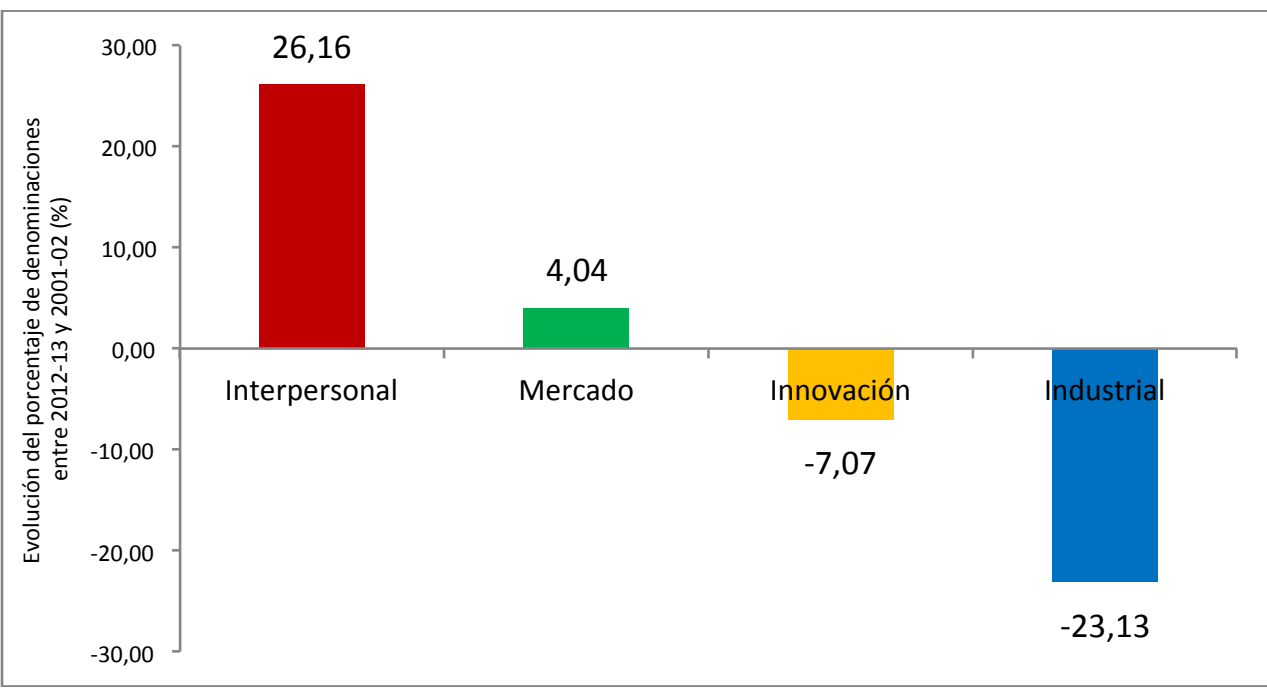

Para conocer en profundidad los cambios de composición registrados entre 2001-02 y 2012-13 es necesario analizar sus 2 factores principales: El mundo de producción de las nuevas denominaciones incorporadas a la clasificación en 2012-13 y la evolución de las denominaciones que han sido clasificadas en ambos cortes temporales, tanto las que han presentado algún tipo de cambio como las que se han mantenido estables.

\section{IV.2.2. Incorporación de nuevas denominaciones}

Pese a las variaciones en la composición que se han identificado, tan solo 15 denominaciones de las 43 clasificadas en ambas campañas han experimentado algún tipo de movimiento entre mundos de producción. Este hecho pone de relieve la importancia de la creación de nuevas denominaciones en la modificación del porcentaje de adscritas a cada mundo de producción (Figura 9). El marco legislativo puesto en marcha en 2003 ha facilitado la creación de denominaciones pequeñas, muy especializadas y orientadas a mercados de calidad. Este hecho explica una parte fundamental del aumento registrado por el mundo interpersonal y de la reducción del peso del mundo industrial (Figura 10).

En 2012-13 se clasifican 23 denominaciones que no habían sido clasificadas en 2001-02, el $65,22 \%$ de ellas ha pasado a integrar el mundo interpersonal. Un 73,91\% de las denominaciones clasificadas en 2012-13 y no en 2001-02 son denominaciones de nueva creación, además un 30,43\% del total son vinos de pago. Estas cifras muestran una tendencia por parte de las nuevas denominaciones a ubicarse dentro del mundo interpersonal, especialmente los pagos vitícolas. Entre ellas pueden citarse los casos de Prado Irache y la mayor de los pagos manchegos. 
Figura 9

NÚMERO DE NUEVAS INCORPORACIONES A LA CLASIFICACIÓN ENTRE 2001-02 Y 2012-13 Y SALIDAS

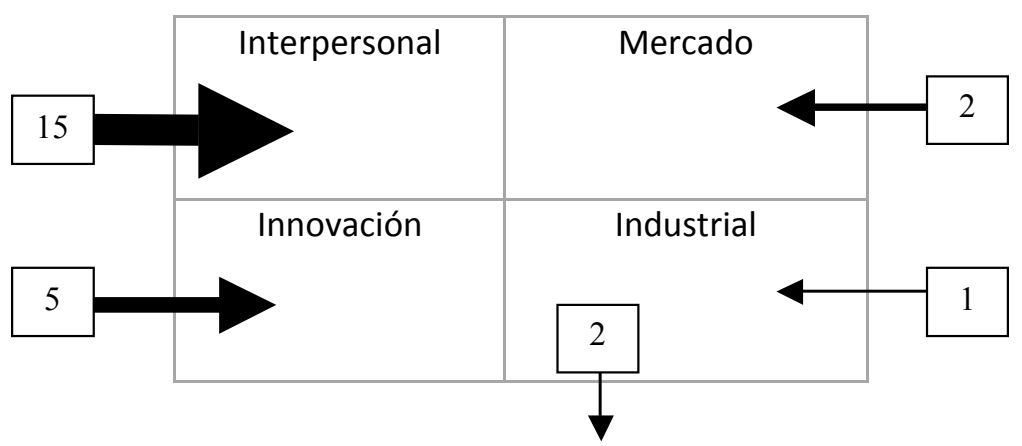

Figura 10

MUNDO DE PRODUCCIÓN DE LAS DENOMINACIONES CLASIFICADAS POR PRIMERA VEZ EN 2012-13
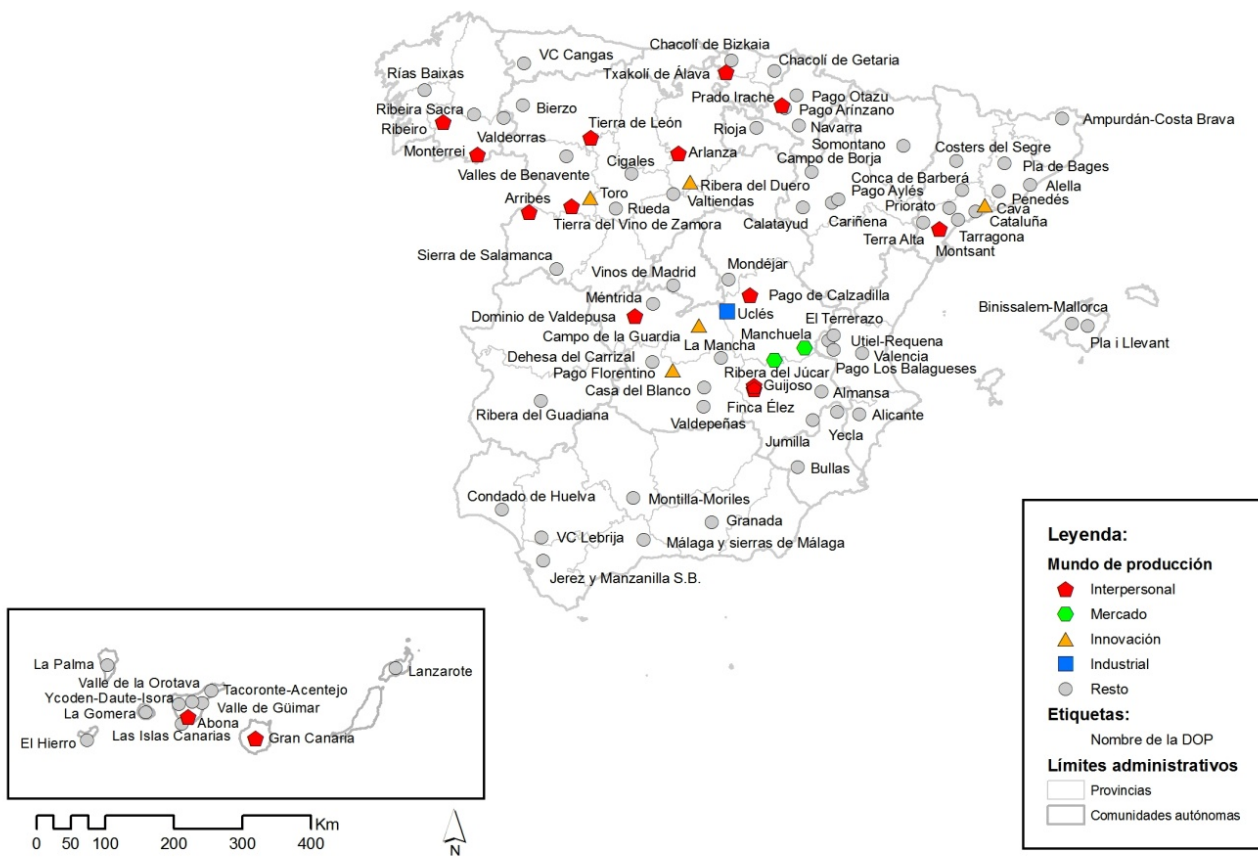
Arribes Toro $\triangle$ Ribera del Duero PagoAylés

Tierra del Vino de Zamora Calatayud cariñena Priorato Cataluña

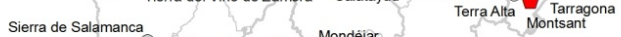

Sierra de Salamanca Vinos de Madrid Mondéjar Montsant

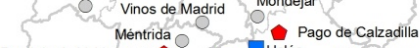

Dominio de Valdepusa Uuclés El Terrerazo Binissalem-Mallorca

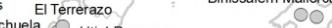

Campo de la Guardia La Mancha Manchuela 8 Utiel-Requena
Valencia
Dehesa del Carrizal
Pago Florentino $\triangle$ Ribera del Júcar Pago Los Balagueses

Ribera del Guadiana

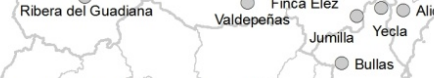

Condado de Huelva Montilla-Moriles

O vCLebrija 1 Málaga y sierras de Málaga

Jerez y Manzanilla S.B.

Jerezy

Leyenda:

Mundo de producción

Interpersonal

- Mercado

$\triangle$ Innovación

Industrial

Resto

Etiquetas:

Nombre de la DOP

Limites administrativos Provinclas

Comunidades autónomas

El aumento de la especialización queda puesto de manifiesto por el gran número de entradas de denominaciones que, junto al mundo interpersonal, ha experimentado el mundo de la innovación. En total 20 denominaciones se han incorporado a alguno de estos mundos de producción, sin embargo, entre las denominaciones que se han clasificado dentro del mundo 
de la innovación por primera vez en 2012-13, algunas como Ribera del Duero o Cava ya se encontraban activas en la campaña de 2001-02. Este aumento del peso de las denominaciones especializadas se contrapone a los mundos de producción estandarizados, donde la relación entradas-salidas se ha mantenido estable, pese a mostrar un ligero crecimiento el mundo mercado.

\section{IV.2.3. Denominaciones estables}

Un $65,12 \%$ de las denominaciones clasificadas en ambos cortes temporales no ha registrado cambios. El mundo de producción donde un mayor número de denominaciones se ha mantenido estable es el mundo industrial (Figura 11), pese a la pérdida de peso relativa que ha experimentado en el periodo de estudio. En total un 63,63\% de las denominaciones ubicadas en el mundo industrial en 2001-02 no ha registrado cambios entre mundos de producción. Se observa que algunas de estas denominaciones han aumentado su puntuación en el indicador de estandarización, entre ellas se puede destacar el caso de Utiel-Requena, Valdepeñas, La Mancha o Cariñena (Figura 12). En estas denominaciones se afianza su posición dentro del mundo industrial, mejorando su capacidad de comercialización de grandes volúmenes de vino y una competencia centrada en el precio. Frente a ellas pueden observarse casos como el de Somontano que, dentro del mundo industrial, ha seguido la tendencia contraria.

Todas las denominaciones clasificadas en el mundo interpersonal en 2001-02 se han mantenido estables. Casos como el de Bierzo que se encontraban cercanos al punto de ruptura en 2001-02 afianzan claramente su posición dentro del mundo interpersonal, a través de una significativa reducción de su puntuación en tipo de tecnología, pasando de 93,18 ha/bodega en el primer corte temporal a 43,1 en el segundo. Por su parte las denominaciones del mundo de la innovación de Rioja, Rías Baixas, Chacolí de Getaria y Montilla-Moriles también han mantenido su mundo de producción. El mundo del mercado ha sido el que ha presentado un menor número de denominaciones estables: Lanzarote y Ribera del Guadiana.

Figura 11

NÚMERO DE DOP CLASIFICADAS EN EL MISMO MUNDO DE PRODUCCIÓN EN 2001-02 Y 2012-13

\begin{tabular}{|c|c|}
\hline Interpersonal: & Mercado: \\
8 & 2 \\
\hline Innovación: & Industrial: \\
4 & 14 \\
\hline
\end{tabular}


Figura 12

MAPA DE DENOMINACIONES QUE NO HAN VARIADO SU MUNDO DE PRODUCCIÓN

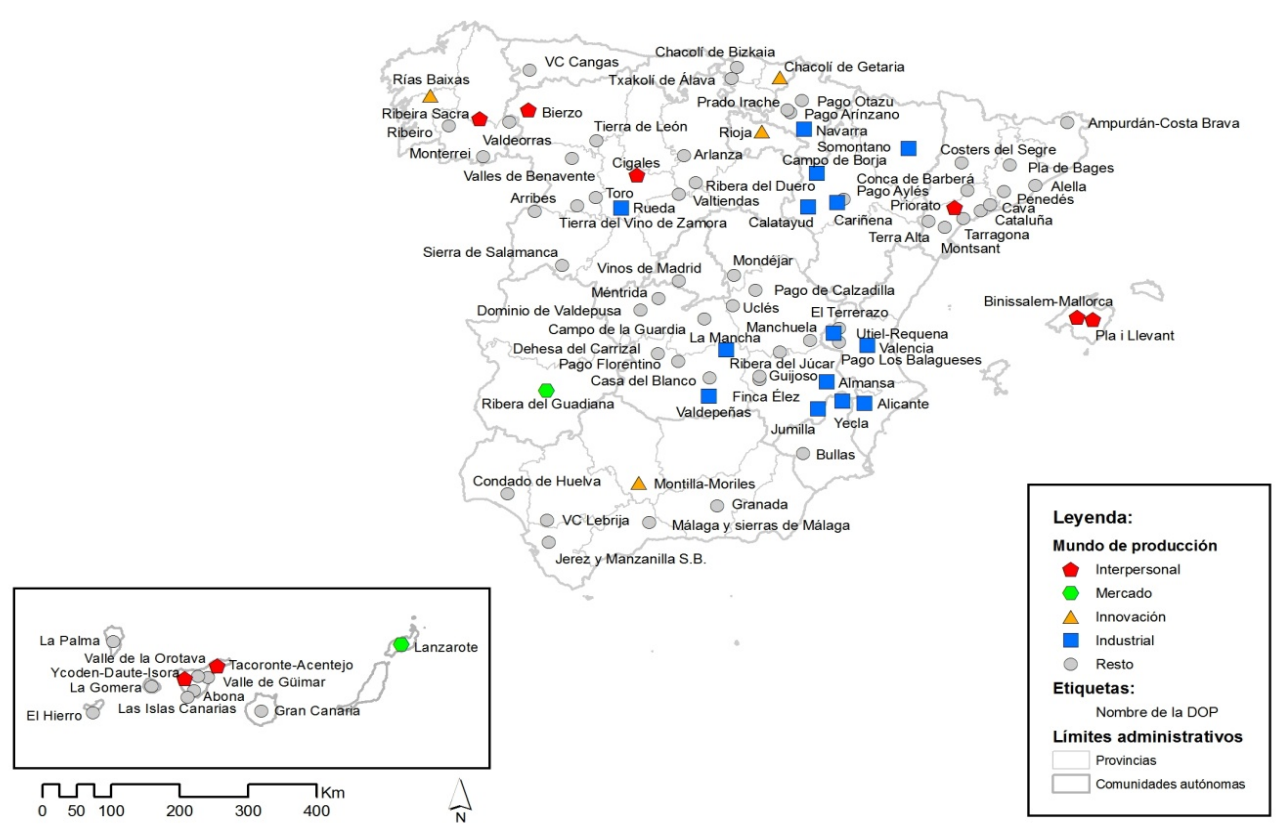

\section{IV.2.4. Movimientos entre mundos de producción}

El 34,88\% de las denominaciones analizadas en 2001-02 y 2013 han registrado cambios de mundo de producción. Los cambios basados en modificaciones de tipo de tecnología tienen un peso menor que los de tipo de mercado: El 73,33\% de movimientos registrados han estado relacionados con un aumento de la dedicación, es decir, las denominaciones que han variado su mundo de producción se han dirigido mayoritariamente a nichos de mercado más específicos, diferenciando su producción. El desplazamiento más frecuente ha tenido como origen el mundo industrial y como destino el mundo del mercado (Figura 13), las denominaciones que han protagonizado este cambio se concentran principalmente en Cataluña (Figura 14), donde puede citarse el caso de Penedés que ha pasado de 378,32 a 145,16 hl/marca.

El mundo interpersonal ha sido el destino de denominaciones procedente de todos los mundos de producción, sin embargo el paso más frecuente ha sido desde el mundo de la innovación. El cambio innovación-interpersonal lo ha realizado un gran número de denominaciones, entre ellas Ampurdán-Costra Brava o Málaga-Sierras de Málaga, que presentan un importante aumento de la dedicación en el eje de mercado. Los cambios en el tipo de tecnología han sido muy poco frecuentes y, salvo el caso de la denominación de Cataluña, de recorridos muy cortos. La denominación de Cataluña, constituida en 2001, puede citarse como claro ejemplo de estandarización, vinculado a procesos propios de la implantación de la denominación, como el aumento de la superficie inscrita. 
Figura 13

DIAGRAMA DE MOVIMIENTOS REGISTRADOS ENTRE 2001-02 Y 2012-13

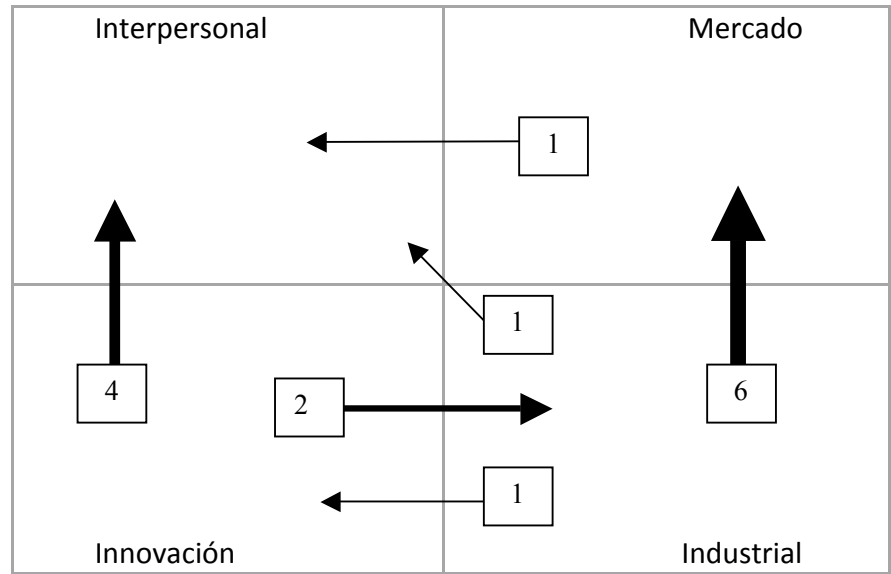

Figura 14

MAPA DE MOVIMIENTOS ENTRE MUNDOS DE PRODUCCIÓN ENTRE 2001-02 Y 2012-13
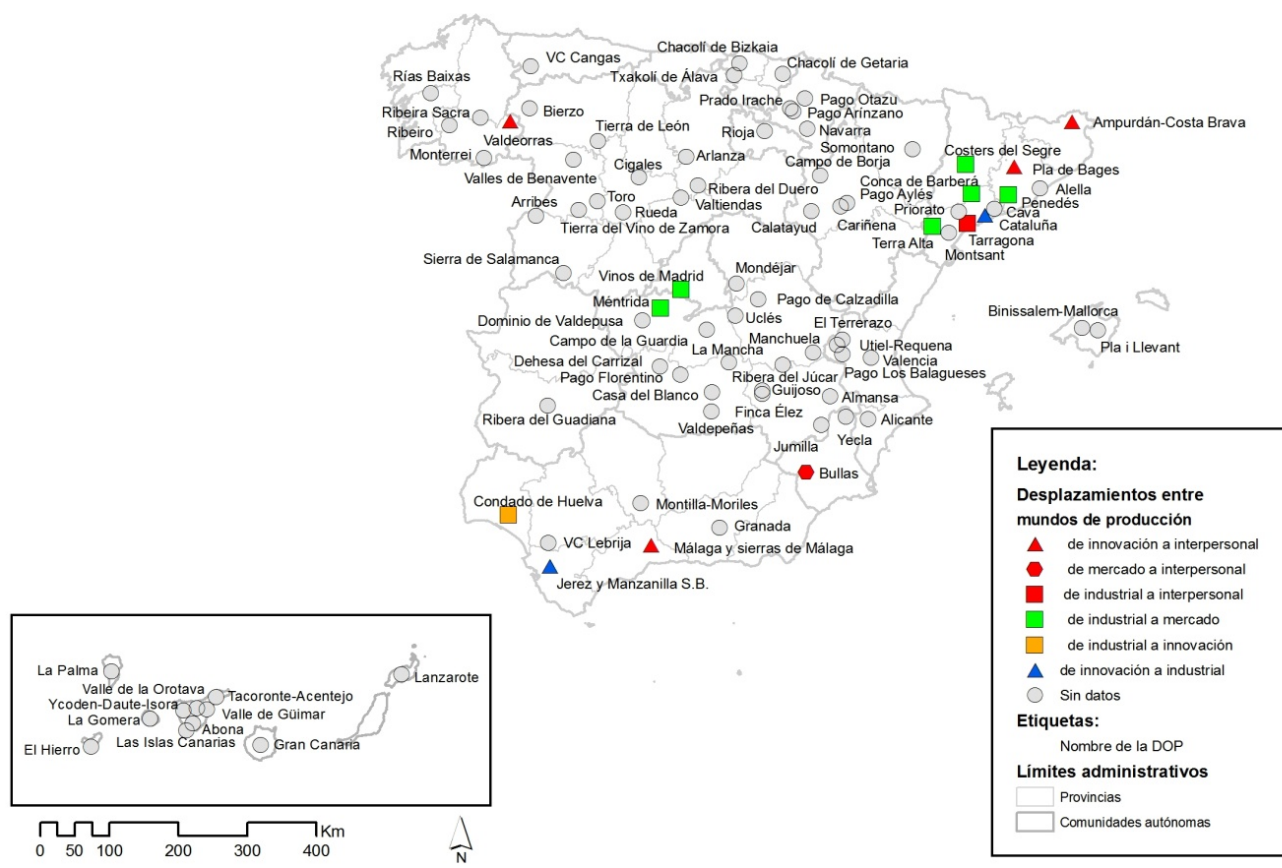


\section{CONCLUSIONES}

La teoría de los mundos de producción se puede utilizar para caracterizar los diferentes tipos de denominaciones de origen españolas de vino adoptando una metodología cuantitativa, como ya plantearon Climent y Sánchez (2015). La utilización de la ratio de superficie por bodega como indicador de tipo de tecnología y el tamaño medio de la marca como indicador de tipo de mercado ha ofrecido unos resultados coherentes con el marco teórico, similares a los que obtuvo Esteban (2016) utilizando un número diferente de campañas. Cada mundo de producción presenta una relación entre precio y calidad diferente: el peso de la competencia en precios es mayor en contextos estandarizados que en los especializados, a su vez el peso de la competencia en calidad diferenciada es mayor en mercados dedicados que en mercados genéricos, tal como se plantea en el marco teórico (Salais y Storper, 1992). Sin embargo sería interesante profundizar en los aspectos relacionados con la delimitación precisa del punto de ruptura, para conseguir una mayor precisión en la clasificación.

En la campaña de 2001-02 se observa un claro predominio de los mercados genéricos, siendo las denominaciones orientadas a mercados personalizados muy poco frecuentes. En esta campaña, el mundo industrial ocupa la mayor parte de la península, destacando denominaciones como La Mancha. En el mismo corte temporal, denominaciones como Rioja, que cuentan con alto grado de reconocimiento de la calidad, se encontraban ya ubicadas dentro del mundo de la innovación. Las denominaciones en mundos de producción con mercados dedicados son muy escasas en 2001-02, pudiendo citarse como ejemplo dentro del mundo interpersonal el caso de los vinos insulares y el de Priorato.

En la campaña de 2012-13, con las figuras introducidas por la ley de la viña y el vino ya operativas, se observan importantes diferencias respecto a 2001-02. Destaca el aumento del número de denominaciones en el mundo interpersonal y el descenso relativo del mundo industrial. La mayor parte de las denominaciones constituidas en el periodo de estudio han pasado a integrar el mundo interpersonal, especialmente los pagos vitícolas. Este hecho confirma el papel de la ley de la viña y el vino en la diversificación del sector.

Las denominaciones clasificadas en el mundo industrial han experimentado pocos cambios de mundo de producción, observándose ajustes internos hacia una mayor estandarización para poder conseguir una mayor competencia en precios. Estas denominaciones preferentemente se encuentran en el centro peninsular, levante y el valle del Ebro. El mundo interpersonal tiene un mayor peso en el noroeste de España, en las islas y en los pagos vitícolas, en estas zonas se observa un mayor número de denominaciones enfocadas a mercados elitistas que permiten que la competencia entre empresas se centre en la calidad. El mundo del mercado se encuentra poco extendido, las compañías que operan en él cuentan con importantes tensiones internas entre diferenciación y competencia en precios. El mundo de la innovación está integrado por denominaciones de gran prestigio como Rioja, Ribera del Duero o Cava, estas denominaciones especializadas cuentan con un tipo de producción capaz de abastecer mercados de gran tamaño en los que la calidad juega un papel importante. En España el número de denominaciones ubicadas en el mundo de la innovación es reducido, pese a que se trate de denominaciones con volúmenes comercializados altos. Estas denomi- 
naciones suponen tan solo el 15,15\% del total en 2012-13, lo que contrasta con el alto peso que tienen en otros países como Italia (Adinolfi et al.,2011).

Se observa una tendencia hacia una mayor dedicación de la producción por parte de un alto porcentaje de denominaciones, estos resultados coinciden con los planteados para el caso de Catilla y León por parte de Sánchez et al. (2010) y Esteban (2016) para el conjunto de España, por otro lado se registra un afianzamiento de las denominaciones industriales. El resto de mundos de producción son poco frecuentes, este hecho presenta un panorama dual entre las denominaciones, existiendo una clara diferenciación de aquellas que tienden a centrar su competencia en calidad de las que tienden a centrarla en la comercialización de grandes volúmenes de vino a precios asequibles.

Como futuras líneas de trabajo e investigación sería interesante mantener actualizada la clasificación, según se genera nueva información. Por otro lado ampliar el periodo de estudio y el ámbito espacial: conocer el sector con una perspectiva temporal más amplia y poder poner en relación las denominaciones españolas con las de otros países, para poder efectuar comparaciones. Así mismo es oportuno incidir en el trabajo de clasificación, de cara a optimizar la elección de los puntos de ruptura de los ejes de clasificación. El sector del vino tiene un importante papel en el desarrollo regional de los espacios rurales y estos análisis pueden utilizarse para conseguir realizar alianzas estratégicas que permitan asentar población y mejorar la calidad de vida en estos territorios.

\section{BIBLIOGRAFÍA}

ADINOLFI, F., DE ROSA, M. y TRABALZI, F. (2011): «Dedicated and generic marketing strategies». British Food Journal, vol. 113 (3), 419-435.

APARICIO, J., SÁNCHEZ, J. L., ALONSO, J. L. y RODERO, V. (2008): «La Ribera del Duero, geografía de un medio innovador en torno a la vitivinicultura». Scripta Nova , $\mathrm{n}^{\circ}$ 277, 1-33.

CAZALS, C. (2012): «Examining the conventions of voluntary environmental approaches in French agriculture». Cambridge Journal of Economics, vol. 36 (5), 1181-1198.

CLIMENT, E. y SÁNCHEZ, J.L. (2015): «La théorie des conventions en géographie économique: un éclairage apporté par l'application à l'industrie espagnole du vin». Géographie, Économie, Société, vol. 17 (1), 25-47.

ESCALONA, A. I., LOSCERTALES, B. y CLIMENT, E. (2013): «Enfoques, experiencias y propuestas para la mejora de la competitividad territorial de las áreas vitivinícolas: las denominaciones de origen protegidas de la provincia de Zaragoza». Boletín de la Asociación de Geógrafos Españoles, $\mathrm{n}^{\circ}$ 62, 317-341.

ESTEBAN, S. y CLIMENT, E. (2015): «Los ‘mundos de producción' del vino en España: un enfoque cuantitativo aplicado a las denominaciones de origen protegidas», en Análisis espacial y representación geográfica: innovación y aplicación (de la Riva, J., Ibarra, P., Montorio, R., Rodriguez, M., Eds.) Zaragoza, Universidad de Zaragoza-AGE, 19952004.

ESTEBAN, S. (2016): Las Denominaciones de Origen Protegidas del sector del vino en España: análisis comparativo desde la teoría de los mundos de producción. Zaragoza, Prensas de la Universidad de Zaragoza. 
HAIR, J., ANDERSON, R., TATHAM, R. y BLACK, W. (1999): Análisis multivariante. Madrid, Prentice Hall Iberia.

KIRWAN, J. (2006): «The Interpersonal World of Direct Marketing: Examining Conventions of Quality at UK farmers' Market». Journal of Rural Studies, vol. 22 (3), 301-312.

LINDKVIST, K. y SÁNCHEZ, J. (2008): «Conventions and Innovation: a Comparison of two Localized Natural Resource-Based Industries». Regional Studies, vol. 43 (2), 343354.

MAGRAMA (2001): Datos de las Denominaciones de Origen protegidas de vinos (DOPS): Campaña 2000 /2001. Madrid, Ministerio de Agricultura, Pesca y Alimentación. Disponible en: http://www.magrama.gob.es/es/alimentacion/temas/calidad-agroalimentaria/ vinos01_02_tcm7-141841.pdf

MAGRAMA (2014): "Datos de las Denominaciones de Origen protegidas de vinos (DOPS): Campaña 2012 /2013”. Madrid, Ministerio de Agricultura, Alimentación y Medio Ambiente. http://www.magrama.gob.es/es/alimentacion/temas/calidad-agroalimentaria/ DATOS_DOPs_VINOS_campa\%C3\%B1a_2012-2013_WEB_(2)_tcm7-329236.pdf

MURDOCH, J. y MIELE, M. (1999): «Back to nature: Changing 'worlds of production' in the food sector». Sociologia Ruralis, vol. 39 (4), 465-483.

MURDOCH, J., MARSDEN, T. y BANKS, J. (2000): «Quality, nature and embeddedness: some theoretical considerations in the context of the food sector». Economic Geography, vol. 76 (2), 107-125.

RUIZ PULPÓN, Á.R. (2013): «Producción agroalimentaria de calidad y postproductivismo agrario: El caso de los vinos de pago en Castilla-La Mancha». Anales de Geografía, $\mathrm{n}^{\circ}$ 33, 137-154.

SALAIS, R. y STORPER, M. (1992): «The four 'Worlds' of Contemporary Industry». Cambridge Journal of Economics, vol.16 (2), 169-193.

SALAZAR, I. y GALVE, C. (2011): «Análisis de la organización interna de la industria: Una aplicación a la industria vitivinícola en la Denominación de Origen Calificada Rioja». Ekonomiaz: Revista vasca de economía, $\mathrm{n}^{\circ} 77,280-300$.

SÁNCHEZ, J., APARICIO, J. y ALONSO, J. (2010): «The Shift between Worlds of Production as an Innovative Process in the Wine Industry in Castile and Leon, Spain» Geoforum, $\mathrm{n}^{\circ} 41,469-478$.

STORPER, M. y SALAIS, R. (1997): Worlds of Production: The Action Frameworks of the Economy. Cambridge, Massachusetts, Harvard University Press.

STRÆTE, E.P. (2004): «Innovation and changing 'worlds of production'. Case-studies of Norwegian dairies». European Urban and Regional Studies, vol. 11 (3), 227-241. 
